THE AsTROPHYSICAL JouRNAL, 517:399-415, 1999 May 20

(c) 1999. The American Astronomical Society. All rights reserved. Printed in U.S.A.

\title{
A COMPARATIVE STUDY OF THE MASS DISTRIBUTION OF EXTREME-ULTRAVIOLET-SELECTED WHITE DWARFS ${ }^{1}$
}

\author{
R. NAPIWOTZKI
}

Dr. Remeis-Sternwarte, Sternwartstrasse 7, 96049 Bamberg, Germany; ai23@sternwarte.uni-erlangen.de

PAUl J. GreEN

Harvard-Smithsonian Center for Astrophysics, 60 Garden Street, Cambridge, MA 02138; pgreen@cfa.harvard.edu

AND

REX A. SAFFER

Department of Astronomy and Astrophysics, Villanova University, 800 Lancaster Avenue, Villanova, PA 19085; saffer@ast.vill.edu Received 1998 June 4; accepted 1998 December 28

\begin{abstract}
We present new determinations of effective temperature, surface gravity, and masses for a sample of 46 hot DA white dwarfs selected from the Extreme Ultraviolet Explorer (EUVE) and ROSAT Wide Field Camera bright source lists in the course of a near-infrared survey for low-mass companions. Our analysis, based on hydrogen non-LTE model atmospheres, provides a map of LTE correction vectors, which allow a thorough comparison with previous LTE studies. We find that previous studies underestimate both the systematic errors and the observational scatter in the determination of white dwarf parameters obtained via fits to model atmospheres. The structure of very hot or low-mass white dwarfs depends sensitively on their history. To compute white dwarf masses, we thus use theoretical mass-radius relations that take into account the complete evolution from the main sequence. We find a peak mass of our white dwarf sample of $0.59 \mathrm{M}_{\odot}$, in agreement with the results of previous analyses. However, we do not confirm a trend of peak mass with temperature reported in two previous analyses. Analogous to other EUV-selected samples, we note a lack of low-mass white dwarfs and a large fraction of massive white dwarfs. Only one white dwarf is likely to have a helium core. While the lack of helium white dwarfs in our sample can be easily understood from their high cooling rate, and therefore low detection probability in our temperature range, this is not enough to explain the large fraction of massive white dwarfs. This feature very likely results from a decreased relative sample volume for low-mass white dwarfs caused by interstellar absorption in EUV-selected samples.
\end{abstract}

Subject headings: binaries: close — stars: atmospheres — stars: evolution — ultraviolet: stars white dwarfs

\section{INTRODUCTION}

UV observations of EUV-detected stars have revealed the presence of about 15 hot white dwarf (WD) companions to bright stars in noninteracting binary systems (see, e.g., Burleigh, Barstow, \& Fleming 1997). At optical wavelengths, these WDs are hidden because of their close proximity to much more luminous companions, which are main-sequence (spectral type K or earlier) or evolved stars.

A fascinating variety of objects are known or proposed to contain WD stars in interacting binary systems. A partial list includes novae, cataclysmic variables, symbiotic stars, $\mathrm{Ba}$ and $\mathrm{CH}$ giants, Feige 24 type systems, and dwarf carbon stars (Green \& Margon 1994). These systems offer great insights to evolution and dynamical processes in binaries.

A number of interacting binary systems where the WD is the primary (i.e., optically brightest) star have also been found among EUV-detected systems (e.g., six close, interacting WD/red dwarf binaries by Vennes \& Thorstensen 1994). Optical or ultraviolet spectral observations are most commonly used to detect companions to WD primaries by searching for (1) the presence of narrow Balmer line emission overlying the broad smooth Balmer absorption of the WD, (2) a composite WD plus main sequence spectrum, or

\footnotetext{
${ }^{1}$ Spectral observations reported here were obtained with the Multiple Mirror Telescope, a joint facility of the University of Arizona and the Smithsonian Institution, and with the Bok telescope at the Steward Observatory of the University of Arizona.
}

(3) radial velocity (RV) variations. However, only WDs with very close or intrinsically active companions will be found by case (1). For hot WD systems, composite spectra (case [2]) are only expected to be visible if the companion's spectral type is early enough. RV variations (case [3]) require multiple observations at high spectral resolution, and detection strongly favors close and/or massive companions.

All of the discoveries mentioned above have been strongly dominated by these selection effects, with companions biased to earlier types than predicted by the simulations of deKool \& Ritter (1993) and others. Scaling from deKool \& Ritter's (1993) results, Vennes \& Thorstensen (1994) estimate that "at least twice as many close binary systems remain to be identified from EUV surveys, most of them with a low-mass secondary." The resulting sample of binaries known to date, therefore, must diverge strongly from the intrinsic distribution, in overall normalization, as well as in mass and spectral type of the main sequence companions.

The current study, conceived as a complement to optical studies, began as a near-IR photometric survey for lowmass companions to hot WDs. By investigating only EUVdetected WDs, we obtain a very reasonably sized but complete sample of young WDs, next to which very latetype dwarf companions can be detected in the near-infrared by searching for a $K$ excess over that expected from the WD. Many hot WDs ( $T_{\text {eff }}>24,000 \mathrm{~K}$; Finley et al. 1993) have been detected in the recent EUV all-sky surveys. EUV 
detection of these hot WDs depends primarily on their temperature, distance, and the intervening Galactic interstellar medium (ISM). Our sample of EUV WDs (whose selection we define below) offers excellent flux contrast in the IR relative to optical; cool companions will almost always be brighter in the $K$ band than the hot WDs.

To know what $K$ magnitude to expect for the WDs, we benefit from constraints on $\log g$, radius, and $T_{\text {eff }}$ derivable from optical spectra for the WDs in our sample using non-LTE (NLTE) model atmosphere fits (Napiwotzki et al. 1993; Napiwotzki 1997). The resulting predictions for $K$ magnitudes allow a direct search for any IR excess from a cool companion. In some cases, IR colors will also provide a preliminary spectral type. Results from the IR survey will be presented in an upcoming paper.

An additional motivation is the study of the WD mass distribution. Since the pioneering work of Koester, Schulz, \& Weidemann (1979, hereafter KSW79), it is well established that the masses of WDs cluster in a narrow range around $0.6 M_{\odot}$, remarkable given that WDs stem from progenitors with masses ranging from below $1 M_{\odot}$ up to $\approx 8 M_{\odot}$. Precise knowledge of the WD mass distribution puts constraints on the theory of stellar evolution, especially the poorly understood mass-loss process during the final stages of stellar evolution. With two recent exceptions (Beauchamp et al. 1996 and Dreizler \& Werner 1996, who analyzed samples of helium-rich DB and DO WDs, respectively), the mass distribution has only been determined for hydrogen-rich DA WDs. However, this is not a severe limitation because this spectral class comprises about $80 \%$ of all known WDs.

The analysis of KSW79, along with other follow-up investigations in the early eighties, used photometric data of which the Strömgren and Greenstein multichannel colors were the most suitable. Both systems provide temperatureand gravity-sensitive indices. Alternatively, KSW79 and others used trigonometric parallax measurements to directly calculate the stellar radius; however, the latter method is practical only for a small sample and suffers from considerable measurement uncertainty. Unfortunately, the photometric indices have their highest sensitivity near $10,000 \mathrm{~K}$. At this temperature, DA WDs have a convective atmosphere, and the results depend critically on the adopted parameters of mixing length theory.

The situation improved at the beginning of the nineties when the development of modern, highly efficient detectors made it possible to obtain high-quality spectra of large numbers of WDs and determine the stellar parameters from a fit to the detailed profiles of the Balmer lines. This method yields sufficient accuracy for WDs hot enough to have a radiative envelope. The first comprehensive sample of WDs analyzed by this method was presented by Bergeron, Saffer, \& Liebert (1992, hereafter BSL92). As attributed to higher precision of spectroscopic methods, this investigation yielded a WD mass distribution even narrower than found by KSW79 and other previous studies.

At the same time, Kidder (1991) analyzed a sample of hot DA WDs discovered through positional coincidences of cataloged hot DA WDs in existing soft X-ray databases. Three soft X-ray sources corresponding to WDs were found having relatively low effective temperatures, $\approx 25,000 \mathrm{~K}$, which were determined independently using complementary optical and UV spectroscopy. Kidder et al. (1992) analyzed an expanded sample to derive photospheric $\mathrm{He}$ abundances for the hotter objects and to establish an effective observational low-temperature threshold for the detection of pure hydrogen DA WDs at soft X-ray wavelengths.

In 1997 three groups (Marsh et al. 1997, hereafter M97; Vennes et al. 1997, hereafter V97; Finley, Koester, \& Basri 1997, hereafter FKB97) published results on the mass distribution of EUV-selected WDs. Due to the selection criterion, these samples contain the hottest WDs $\left(T_{\text {eff }}>\right.$ $25,000 \mathrm{~K}$ ), as cooler WDs do not emit significant EUV radiation. The derived mass distributions in the EUVselected samples are similar to that of BSL92 but show some interesting deviations in detail. The frequency of very high mass WDs is much larger, and that of very low mass WDs much smaller, than in BSL92. These findings can at least partly be explained by selection effects (see the discussion in FKB97). More serious is a trend of the peak mass with temperature. V97 found that their mass distribution peaks at $0.598 M_{\odot}$, while the BSL92 distribution peaks at $0.568 M_{\odot}$, with masses computed using Wood's (1995) mass-radius relation with " thick" layers $\left(M_{\mathrm{H}}=10^{-4} M_{\mathrm{WD}}\right.$, $\left.M_{\mathrm{He}}=10^{-2} M_{\mathrm{WD}}\right)$. This discrepancy diminishes slightly if the "very thin layer" $\left(M_{\mathrm{He}}=10^{-4} M_{\mathrm{WD}}\right.$, no hydrogen layer) mass-radius relations are used (peak masses of 0.556 and $0.532 M_{\odot}$ for the V97 and BSL92 samples, respectively). V97 interpreted this as evidence for a very thin hydrogen layer of the DA WDs. However, the effects are small, so this result depends strongly on the accuracy of the derived stellar parameters.

FKB97 estimated the internal accuracy of different analysis methods from Monte Carlo simulations. The precision reachable by Balmer line fitting is very compelling: $\Delta T_{\text {eff }} / T_{\text {eff }}<0.01$ for $T_{\text {eff }}<60,000 \mathrm{~K}$. However, for spectra with very high signal-to-noise ratios $(\mathrm{S} / \mathrm{N})$, errors introduced by details of the observation and reduction techniques (e.g., extraction, flat fielding, flux, and wavelength calibration) might be more important but are very difficult to determine. Additionally, one must take into account differences in the model atmosphere calculations and fitting procedure. Together with the results presented in this paper, we now have four samples of hot WDs analyzed in a similar way and with significant overlap. This offers the opportunity to determine the real accuracy of the spectral analysis of hot WDs, including many possible systematic effects.

We present the selection criteria of our sample in $\S 2$ and the observations and data reduction procedures in $\S 3$. Details on our model atmospheres are given in $\S 4$. The results and a detailed comparison with the previous analyses of EUV-selected WDs are presented in $\S 5$. We finish with a discussion of our results and an outlook.

\section{SAMPLE SELECTION CRITERIA}

We chose to limit our uniform sample to DA's, for which models provide the best temperature and mass constraints. We start with 73 known DA WDs in the Extreme Ultraviolet Explorer (EUVE) bright source list (Malina et al. 1994). Excluding sources at low galactic latitudes $(|b|<15)$ and southerly declinations $(\delta<-20)$ yields a list of 28 DA's. A similar procedure for nonoverlapping DA's listed in the ROSAT Wide Field Camera survey Bright Source Catalogue (Pounds et al. 1993) yields 29 objects.

We have removed from our uniform sample two wellknown stars with published sensitive optical spectrophotometry and IR photometry (Feige 24 and HZ 43). Two 
TABLE 1

Atmospheric Parameters of Program Stars

\begin{tabular}{|c|c|c|c|c|c|}
\hline RE J & Other Names & $T_{\text {eff }}$ & $\log g$ & $M / M_{\odot}$ & Observation Date \\
\hline $0007+331$ & GD 2 & $46493 \pm 514$ & $7.83 \pm 0.05$ & 0.602 & 1996 Jan 6 \\
\hline $0134-160^{a}$ & GD 984, PHL 1043 & $44866 \pm 667$ & $7.77 \pm 0.05$ & 0.572 & 1996 Jan 5 \\
\hline $0237-122$ & PHL 1400 & $32077 \pm 177$ & $8.45 \pm 0.04$ & 0.890 & 1996 Jan 5 \\
\hline $0348-005$ & GD 50 & $3950 \pm 8464$ & $9.07 \pm 0.06$ & 1.215 & 1996 Jan 4 \\
\hline $0427+740$ & & $48587 \pm 1044$ & $7.93 \pm 0.08$ & 0.646 & 1996 Jan 5 \\
\hline $0457-280$ & MCT $0455-2812$ & $51199 \pm 786$ & $7.72 \pm 0.05$ & 0.570 & 1996 Jan 4 \\
\hline $0512-004$ & & $31733 \pm 139$ & $7.40 \pm 0.03$ & 0.458 & 1996 Jan 5 \\
\hline $0521-102$ & & $33186 \pm 301$ & $8.60 \pm 0.06$ & 0.980 & 1996 Jan 5 \\
\hline $0841+032$ & & $38293 \pm 252$ & $7.75 \pm 0.03$ & 0.544 & 1996 Jan 4 \\
\hline $0902-040$ & & $23218 \pm 160$ & $7.84 \pm 0.02$ & 0.544 & 1996 Jan 4 \\
\hline $0907+505$ & PG $0904+511$ & $32167 \pm 338$ & $8.11 \pm 0.07$ & 0.695 & 1996 Jan 4 \\
\hline $0940+502$ & PG $0937+506$ & $36034 \pm 283$ & $7.69 \pm 0.04$ & 0.519 & 1997 Apr 9 \\
\hline $0957+852$ & & $51311 \pm 1348$ & $8.37 \pm 0.10$ & 0.866 & 1996 Jan 5 \\
\hline $1019-140$ & & $31524 \pm 102$ & $7.92 \pm 0.02$ & 0.606 & 1997 Apr 9 \\
\hline $1029+450$ & PG $1026+454$ & $35518 \pm 247$ & $7.70 \pm 0.04$ & 0.520 & 1997 Apr 9 \\
\hline $1032+532$ & & $43587 \pm 506$ & $7.95 \pm 0.05$ & 0.644 & 1996 Jan 4 \\
\hline $1033-114$ & G 162-66, LTT 3870 & $24685 \pm 252$ & $7.85 \pm 0.03$ & 0.553 & 1996 Jan 4 \\
\hline $1036+460^{e}$ & GD 123 & $29361 \pm 251$ & $8.02 \pm 0.05$ & 0.648 & 1996 Jan 4 \\
\hline $1043+490^{\mathrm{e}}$ & & $41132 \pm 1178$ & $7.94 \pm 0.13$ & 0.635 & 1996 Jan 5 \\
\hline $1044+574$ & PG $1041+580$ & $30338 \pm 153$ & $7.81 \pm 0.03$ & 0.550 & 1996 Jan 4 \\
\hline $1100+713$ & PG $1057+719$ & $41104 \pm 814$ & $7.84 \pm 0.09$ & 0.593 & 1996 Jan 4 \\
\hline $1112+240$ & Ton 61 & $39824 \pm 636$ & $7.78 \pm 0.07$ & 0.563 & 1996 Jan 5 \\
\hline $1122+434$ & PG $1120+439$ & $26996 \pm 151$ & $8.31 \pm 0.02$ & 0.803 & 1997 Apr 9 \\
\hline $1126+183^{e}$ & PG $1123+189$ & $54334 \pm 1983$ & $7.76 \pm 0.13$ & 0.594 & 1996 Jan 4 \\
\hline $1128-025$ & PG $1125-026$ & $30699 \pm 380$ & $8.24 \pm 0.08$ & 0.767 & 1996 Jan 4 \\
\hline $1148+183$ & PG $1145+188$ & $25758 \pm 299$ & $7.91 \pm 0.04$ & 0.587 & 1996 Jan 4 \\
\hline $1235+233^{b}$ & PG $1232+238$ & $46569 \pm 523$ & $7.83 \pm 0.05$ & 0.602 & 1996 May $11-12$ \\
\hline $1257+220^{b}$ & GD 153 & $38926 \pm 142$ & $7.78 \pm 0.02$ & 0.560 & 1996 Jan 4, 1996 May 12 \\
\hline $1336+694$ & PG $1335+701$ & $29607 \pm 87$ & $8.34 \pm 0.02$ & 0.824 & 1996 May 10 \\
\hline $1431+370$ & GD 336 & $34404 \pm 115$ & $7.91 \pm 0.02$ & 0.608 & 1996 May 11 \\
\hline $1446+632$ & & $37947 \pm 254$ & $7.79 \pm 0.04$ & 0.562 & 1996 May 11 \\
\hline $1629+780^{c}$ & & $41043 \pm 338$ & $7.92 \pm 0.04$ & 0.627 & 1996 May 11 \\
\hline $1638+350$ & PG $1636+351$ & $35404 \pm 142$ & $7.98 \pm 0.02$ & 0.642 & 1996 May 10 \\
\hline $1643+411^{b}$ & PG $1642+414$ & $28815 \pm 81$ & $8.22 \pm 0.02$ & 0.753 & 1996 May 9 \\
\hline $1650+403^{b}$ & & $38144 \pm 211$ & $7.97 \pm 0.03$ & 0.643 & 1996 May 9 \\
\hline $1711+664^{\mathrm{d}}$ & & $48989 \pm 757$ & $8.89 \pm 0.06$ & 1.141 & 1996 May 12, 1997 Apr 9 \\
\hline $1726+583^{b}$ & PG $1725+586$ & $53561 \pm 542$ & $8.23 \pm 0.04$ & 0.795 & 1996 May 9 \\
\hline $1800+683$ & KUV $18004+6836$ & $44723 \pm 424$ & $7.80 \pm 0.04$ & 0.585 & 1996 May 10 \\
\hline $1820+580$ & & $44099 \pm 264$ & $7.78 \pm 0.03$ & 0.574 & 1996 May 9 \\
\hline $1845+682$ & KUV $18453+6819$ & $36120 \pm 189$ & $8.23 \pm 0.03$ & 0.770 & 1996 May 10 \\
\hline $2116+735^{b}$ & KUV $21168+7338$ & $50812 \pm 354$ & $7.72 \pm 0.03$ & 0.569 & 1996 Jan 5, 1996 May 9 \\
\hline $2207+252$ & & $26964 \pm 174$ & $8.27 \pm 0.03$ & 0.779 & 1996 Jan 5 \\
\hline $2312+104$ & GD 246 & $53088 \pm 968$ & $7.85 \pm 0.07$ & 0.624 & 1996 Jan 4 \\
\hline
\end{tabular}

NoTE.-Error estimates were taken from the $\chi^{2}$ procedure.

${ }^{a}$ Spectrum slightly contaminated by cool companion; the cores of $\mathrm{H} \beta$ and $\mathrm{H} \gamma$ are excluded from the fit.

${ }^{b}$ Weighted mean of individual observations.

${ }^{c}$ Red part of spectrum contaminated by cool companion; $\mathrm{H} \beta$ and the core of $\mathrm{H} \gamma$ excluded from fit.

${ }^{\mathrm{d}}$ Close-by optical companion; spectrum apparently not contaminated.

${ }^{e}$ Red part of spectrum contaminated by cool companion; $\mathrm{H} \beta$ excluded from fit.

DA's with broad-line profiles due to magnetic splitting were also excluded (PG 1658+441 and PG 0136+251). Eight known binaries are also excluded from the uniform sample: V471 Tau (Vennes, Christian, \& Thorstensen 1998), PG $0824+289$ (Heber et al. 1993), HD 74389B (Liebert, Bergeron, \& Saffer 1990), RE J1016 - 052 (V97), PG 1033 + 464 (GD 123; Green, Schmidt, \& Liebert 1986), RE J1426+500 (V97), RE J1629+780 (Catalan et al. 1995), and IK Peg (Wonnacott, Kellett, \& Stickland 1993), leaving 47 objects.

In this paper, a handful of objects that fell outside the uniform sample definitions just outlined were included for observation. These include the known binaries PG $1033+464$ and RE J1629+780, and the magnetic WD PG
$1658+441$, as well as MCT $0455-2812$, which was outside the sample declination limits.

Due to observing constraints (a combination of weather, poor seeing, and faint objects, or celestial placement of objects), no spectra were obtained for sample objects RE J0443-034, RE J0916-194, or PG $1040+451$. PG $1234+482$ was originally classified as an sdB star and thus excluded; it has since been reclassified as a DA (Jordan, Heber, \& Weidemann 1991). The final sample we analyze here thus includes 46 DA WD stars for which we present new model NLTE fits to optical spectra.

We note that since the sample selection was performed, several relevant discoveries pertaining to sample objects 

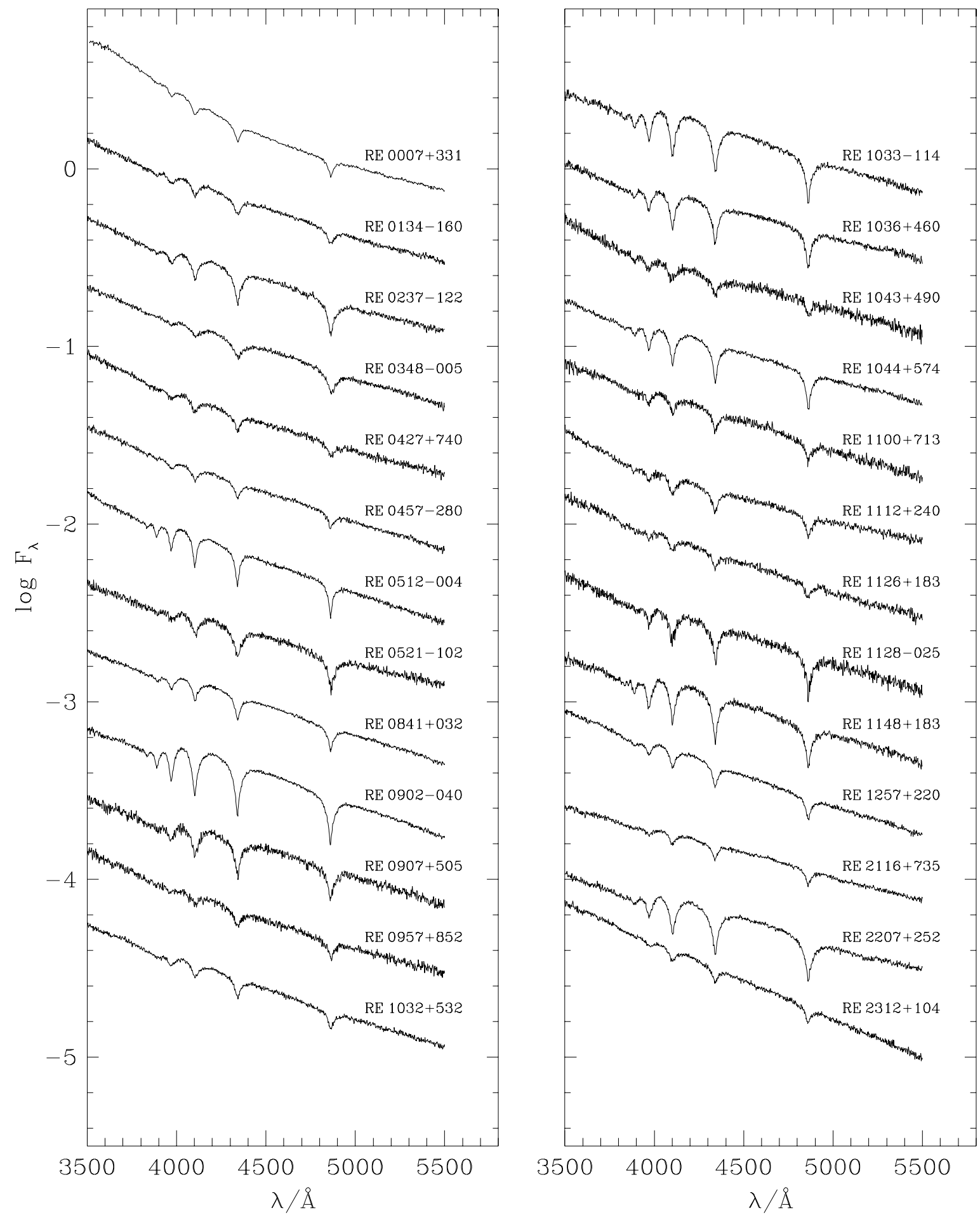

FIG. 1.-Spectra of the WDs observed at Steward Observatory

have been made. RE J0134-160 (GD 984) has central Balmer emission components produced by a dMe companion. RE $\mathrm{J} 1440+750$ turns out to be a magnetic DA (Dreizler et al. 1994). The uniform sample will be discussed in a follow-up paper treating the IR photometry and binary fraction.

\section{OBSERVATIONS}

Dates are listed for all observations in Table 1. On the nights of 1996 January 4-6, we obtained spectra at Steward
Observatory's Kitt Peak Station using the Bok $2.3 \mathrm{~m}$ reflector equipped with the Boller \& Chivens Cassegrain spectrograph and UV-flooded Loral $800 \times 1200$ CCD. Most spectra were dispersed with a 600 line $\mathrm{mm}^{-1}$ first-order grating used behind a 4 ". $5 \times 4^{\prime}$ long slit. The instrumentation provided wavelength coverage $\lambda \lambda 3400-5600$ at a spectral resolution of $\sim 5 \AA$ FWHM. On the last night of the observing run, we employed a new 400 line $\mathrm{mm}^{-1}$ grating providing wavelength coverage $\lambda \lambda 3500-6790$ at a spectral resolution of $\sim 7 \AA$ FWHM. 

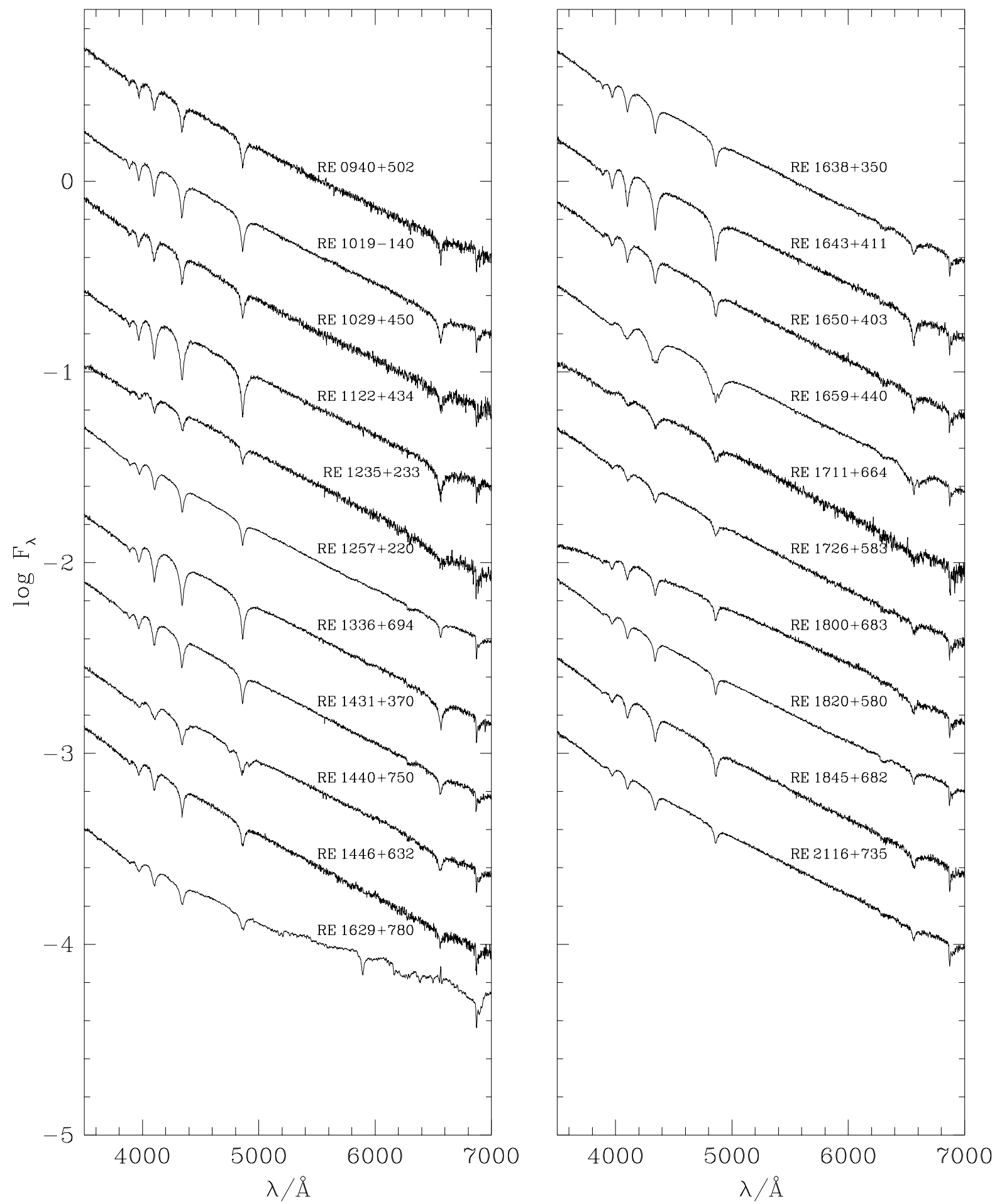

Fig. 2.- Spectra of the MMT sample

We also obtained spectra at the Multiple Mirror Telescope (MMT) on Mt. Hopkins 1996 May 9-11 using the 300 line $\mathrm{mm}^{-1}$ grating in first order on the Loral $3 \mathrm{k} \times 1 \mathrm{k}$ $\mathrm{CCD}$ of the blue channel spectrograph. This yields coverage from about 3350 to $8800 \AA$, and the $2^{\prime \prime}$ slit width we used resulted in a spectral resolution of about $4 \AA$ FWHM. Several objects were kindly obtained for us using the identical instrumental configuration at the MMT by P. Berlind on 1997 April 8.

Exposures at both telescopes ranged from 1 to 30 minutes for program stars, and for all observations the long slit was rotated to the parallactic angle according to the calculations of Filippenko (1982). The air masses were held below 1.5 in almost all cases. All spectra were extracted from the two-dimensional images and reduced to linear wavelength and intensity scales using standard reduction packages in the Image Reduction and Analysis Facility (IRAF). These operations included bias subtraction, flat-field division by images obtained by exposing on dome or internal quartz lamps, centroiding and summation of the stellar traces on the two-dimensional images, sky subtraction, wavelength calibration using spectra of $\mathrm{He} / \mathrm{Ar}$ arc lamps, and absolute 


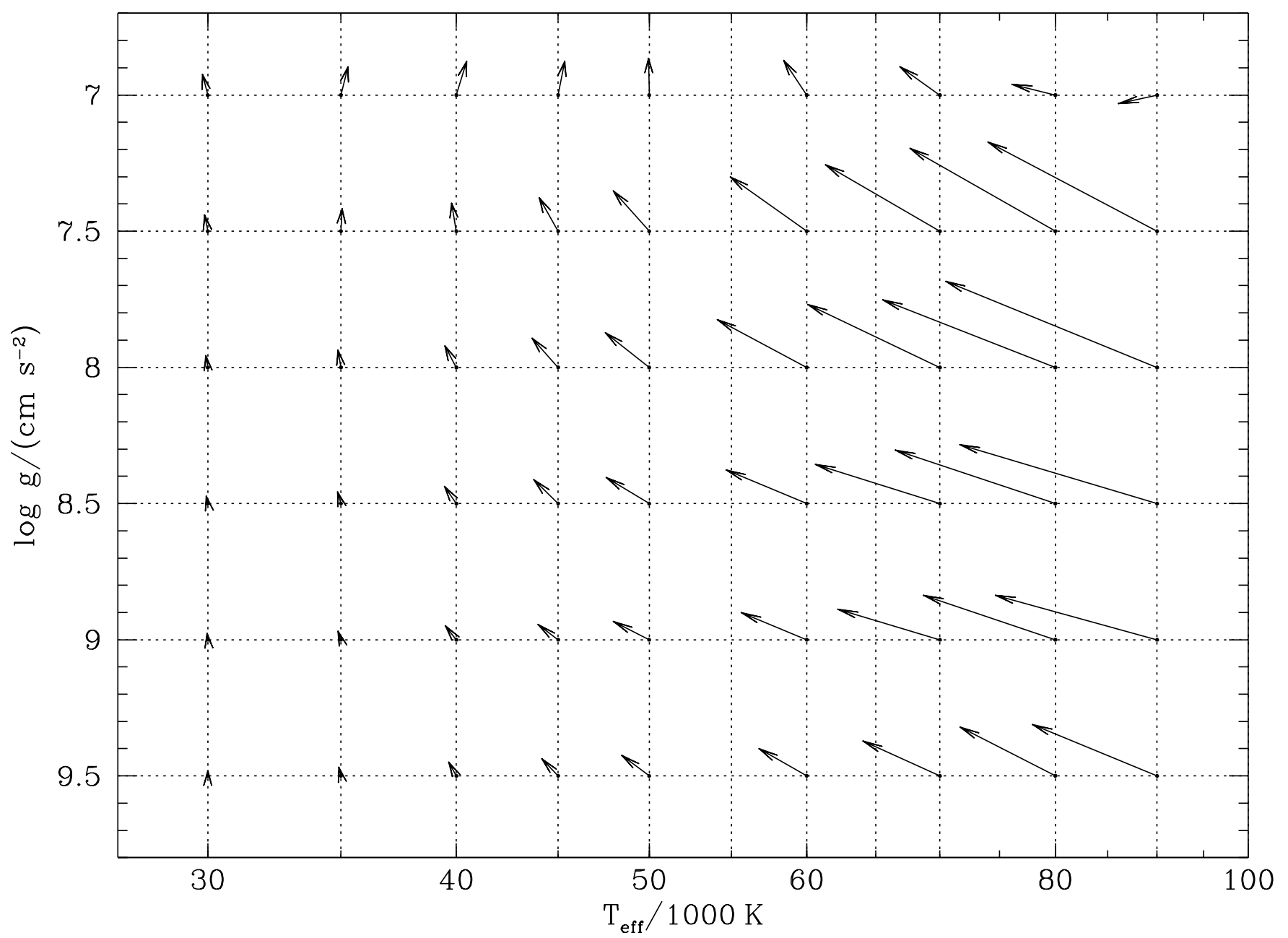

Fig. 3.-LTE offsets. The differences are magnified 3 times. The vectors give the correction that must be applied to transform LTE results to the NLTE scale.

flux calibration using the flux standards of Massey et al. (1988). Details of these reduction procedures are given by BSL92.

We present in Figures 1 and 2 our collection of WD spectra. The spectra obtained at the Steward Observatory $2.3 \mathrm{~m}$ telescope are found in Figure 1, and the MMT spectra are in Figure 2. Some stars observed repeatedly appear in both figures. The $\mathrm{S} / \mathrm{N}$ of our spectra ranges from 35 to 200 , with an average of 90 . Ninety percent of our spectra have an $\mathrm{S} / \mathrm{N}$ of at least 45 .

\section{MODEL ATMOSPHERES}

We calculate hydrogen model atmospheres with the NLTE code developed by Werner (1986). Basic assumptions are those of static, plane-parallel atmospheres in hydrostatic and radiative equilibrium. In contrast to the atmospheres commonly used to analyze DA WDs, we relax the assumption of LTE and solve the detailed statistical equilibrium instead. As described in Werner (1986), the accelerated lambda iteration (ALI) method is used to solve the set of nonlinear equations. The impact of NLTE on WD atmospheres is discussed in detail in Napiwotzki (1997).

$\mathrm{H}$ I levels and lines are included in NLTE up to $n=16$. Line blanketing by the Stark-broadened hydrogen lines is taken into account consistently. As the hydrogen atmospheres of DA WDs are stable for $T_{\text {eff }}>15000 \mathrm{~K}$, convec- tion is not included in our atmospheric models. Pressure dissolution of the higher levels is described by the Hummer \& Mihalas (1988) occupation probability formalism following the NLTE implementation by Hubeny, Hummer, \& Lanz (1994). The synthetic spectra are computed with the extended VCS broadening tables (Vidal, Cooper, \& Smith 1970) provided by Schöning \& Butler (1989, private communication) and Lemke (1997). We followed the prescription of Bergeron (1993) and increased the critical ionizing field adopted to calculate the occupation probability by a factor of 2 . The motivation is not a flaw in the Hummer \& Mihalas (1988) formalism, but a compensation for the inadequacy of the standard Stark broadening theory when line wings overlap. Our NLTE model grid covers the temperature range $17,000 \mathrm{~K}<T_{\text {eff }}<100,000 \mathrm{~K}$ (stepsize increasing with $T_{\text {eff }}$ from 2000 to $10,000 \mathrm{~K}$ ) and gravity range $6.50<\log g<9.75$ (stepsize 0.25 ).

Although deviations from LTE are small for most DA WDs, they become significant for the hottest stars in our sample (see, e.g., Napiwotzki 1997). Since we intend to compare our results with three other samples analyzed by means of LTE atmospheres, we have produced a map with LTE correction vectors. For this purpose we calculated a set of LTE model atmospheres using the technique described in Napiwotzki (1997) of drastically enhancing collisional rates between the atomic levels in the NLTE code. 
This forces the occupation numbers to be in LTE and guarantees consistency with the NLTE atmospheres.

The synthetic LTE spectra were transformed into "observed" spectra by convolving them with a Gaussian of $5 \AA$ FWHM, rebinning them to $2 \AA$, and adding Poisson noise corresponding to a continuum $\mathrm{S} / \mathrm{N}$ of 100 . These simulated spectra were analyzed with the NLTE grid following the procedure outlined in $\S 5$. One thousand simulations were run for every parameter set to eliminate the effect of random errors. The resulting offsets are displayed in Figure 3. The orientation of the vectors corresponds to the correction, which must be applied to transform LTE results to the NLTE scale.

As expected from the results of Napiwotzki (1997), the differences are negligible for DA WDs cooler than $\approx 40,000$ $\mathrm{K}$, but can be significant for hotter stars. The LTE vectors show the trend that NLTE effects increase with increasing temperature and decreasing gravity (see, e.g., Figs. 3 and 4 in Napiwotzki 1997 for the case of DA WDs). The small corrections found for the models with the highest temperature and lowest gravity seems to contradict this behavior. A look at the line profiles reveals that the NLTE deviations are larger for, say, the $T_{\text {eff }}=90,000 \mathrm{~K}$, $\log g=7.0$ model than the $70,000 \mathrm{~K}, 8.0$ model. However, the correction vectors are not a simple function of NLTE deviations measured, e.g., as equivalent width difference, but depend also on the way line profiles vary with temperature and gravity. The small corrections found for the high temperature/low gravity models are produced by the cancellation of these effects. We expect a reliable transformation between the LTE and NLTE temperature scales only if the deviations are not too large. A conservative upper limit is $\approx 70,000 \mathrm{~K}$. A thorough comparison with the previous LTE analyses is presented in $\S 5.3$.

We checked our models by a comparison of our LTE spectra with some DA model spectra kindly provided by D. Koester (1998, private communication). Model parameters were $T_{\text {eff }}=30,000-70,000 \mathrm{~K}$ in $10,000 \mathrm{~K}$ steps, and $\log g=8.0$ for pure hydrogen models. Input physics are very similar. In particular, both model calculations adopt twice the critical ionizing field for the calculation of line profiles. We treated D. Koester's model spectra the same way we treated the LTE spectra above and fitted them with our LTE grid. The result was quite satisfactory: the temperature differences were always below $1.5 \%$, and the gravity differences never exceeded 0.03 dex.

Metals were ignored in our calculations, but they can modify the hydrogen line profiles by their effect on the atmospheric structure. Lanz et al. (1996) analyzed the Balmer lines of the hot DA G191 B2B with pure hydrogen LTE and NLTE atmospheres and an NLTE model with full metal line blanketing. They concluded that the effect of metal line blanketing on the Balmer lines was relatively small, and the difference between LTE and NLTE was found to be the most important effect. A recent study by Barstow, Hubeny, \& Holberg (1998), which investigated several hot WDs in the temperature range around $60,000 \mathrm{~K}$, derived larger metal line blanketing effects of the order of the NLTE effects. Since the LTE analyses of M97, V97, and FKB97 are based on pure hydrogen models, our results should be consistent with theirs in any case.

\section{SPECTRAL ANALYSIS AND RESULTS}

Atmospheric parameters of our DA WDs are obtained by simultaneously fitting line profiles of the observed Balmer lines with the NLTE model spectra described above. We use the least-square algorithm described in BSL92. The observed and theoretical Balmer line profiles are normalized to a linear continuum (both spectra are $F_{\lambda}$ ) in a consistent manner. Wavelength shifts are determined with a cross-correlation method and applied consistently to each complete spectrum. The synthetic spectra are convolved to the observational resolution with a Gaussian and interpolated to the actual parameters with bicubic splines and interpolated to the observed wavelength scale.

The atmospheric parameters $T_{\text {eff }}$ and $\log g$ are then determined by minimizing the $\chi^{2}$ value by means of a LevenbergMarquardt steepest descent algorithm (Press et al. 1986). Several tests revealed that our interpolation routine is rather robust concerning spacing of our model grid and yields reliable results even at the edge of the model grid.

Finally, an estimate of the internal errors can be derived from the covariance matrix. In contrast to BSL92, we estimate the noise of the spectra $(\sigma)$ used for the $\chi^{2}$ fit from the neighboring continuum of each line. The $\mathrm{S} / \mathrm{N}$ is adopted to be constant throughout the line.

The results are given in Table 1, with illustrative examples shown in Figure 4. We adopt, for the moment, the usual practice and indicate in Table 1 the internal errors estimated from the quality of the $\chi^{2}$ fit. However, one should keep in mind that these errors can only serve as lower limits. We will show below ( $\$ 5.3)$ that these formal errors derived from the $\chi^{2}$ fit significantly underestimate the real errors.

External errors can be estimated from multiple observations and analysis of the same star. We obtained repeat observations for a subsample of six stars, for which results are given in Table 2. The gravity values of all six stars agree within the estimated internal errors. The same is also true for four temperature comparisons. However, the differences found for RE J1650+403 and RE J2116+735 are significantly larger. This is in line with the external errors we estimate from a comparison with the studies of M97, V97, and FKB97 (see § 5.3).

\subsection{Binaries}

Five stars in our sample, RE J0134-160, RE $\mathrm{J} 1036+460$, RE $\mathrm{J} 1043+490$, RE $\mathrm{J} 1126+183$, and RE $\mathrm{J} 1629+780$, show clear signs of binarity in the red part of their spectra. Two more stars, RE J1711+664 and RE $\mathrm{J} 2207+502$, are members of visual binaries.

$R E J 1629+780$.- The red part of the spectrum of RE $\mathrm{J} 1629+780$ is heavily contaminated by an M-type mainsequence companion. The composite spectrum and the spectrum of the $M$ star after subtracting the WD component are shown in Figure 5. The characteristic bands of TiO are easily recognizable. Catalán et al. (1995) determined a spectral type of dM4. The $\mathrm{H} \alpha$ and $\mathrm{H} \beta$ lines are seen in emission, which indicates a chromospherically active $\mathrm{Me}$ star. Sion et al. (1995) detected a flarelike increase of the Balmer line emission.

The comparison in Figure 5 demonstrates that the blue part $(\lambda<4400 \AA)$ of the WD spectrum is not disturbed by the $\mathrm{M}$ dwarf. Thus we excluded $\mathrm{H} \beta$ and the core of $\mathrm{H} \gamma$ from the fit and derived the parameters $T_{\text {eff }}=41,000 \mathrm{~K}$ and $\log$ $g=7.92$ from $\mathrm{H} \gamma$ to $\mathrm{H} \eta$. These parameters are in reasonable agreement with the results of Catalán et al. (1995; $T_{\text {eff }}=41,800 \mathrm{~K} ; \log g=8.0$ ), who, however, included $\mathrm{H} \beta$, and Kidder (1991; $\left.T_{\text {eff }}=42,500 \mathrm{~K}, \log g=7.6\right)$, who fitted Ly $\alpha$ and the Balmer lines $\mathrm{H} \beta$ and $\mathrm{H} \gamma$. 


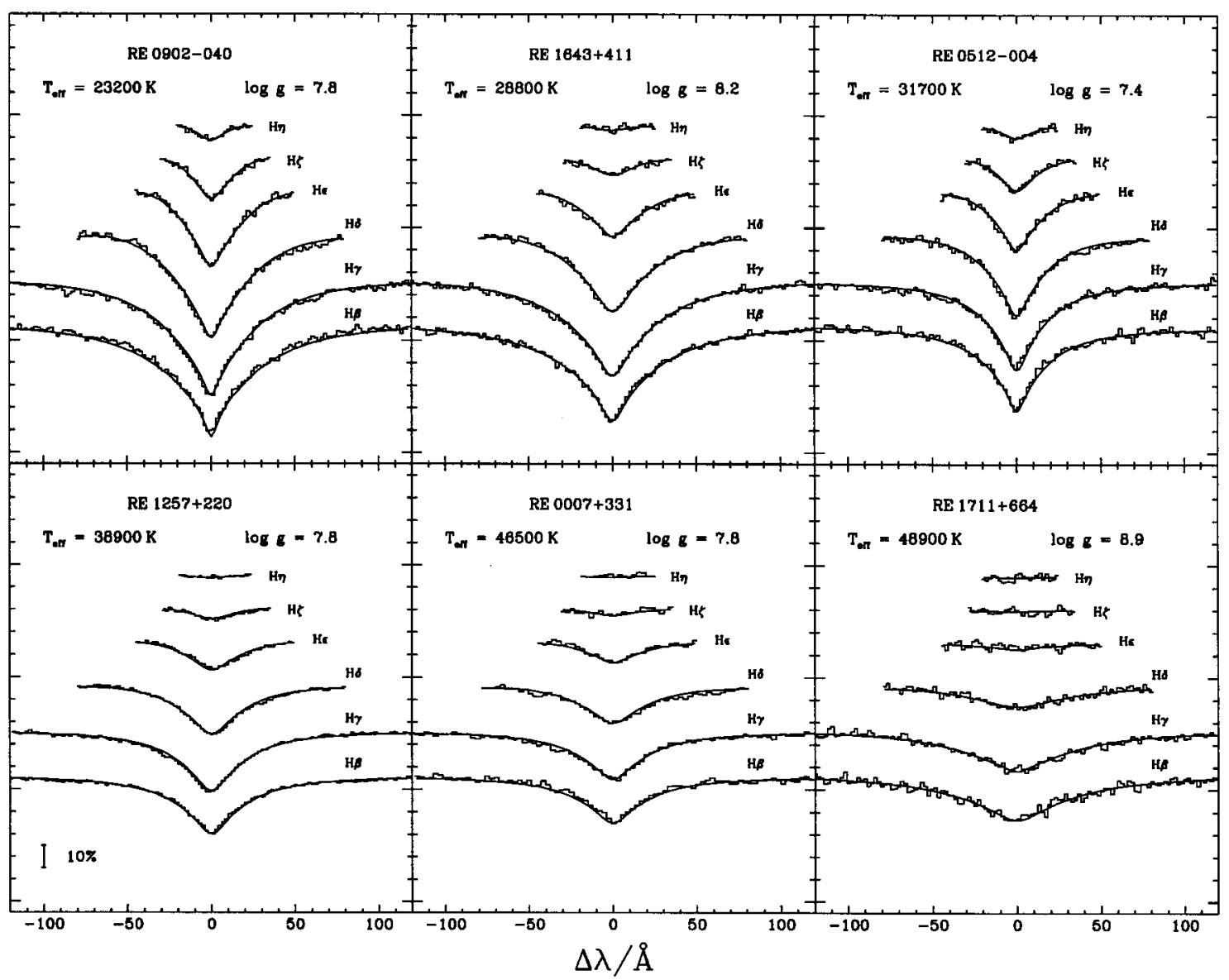

FIG. 4.-Balmer line fits for a representative set of WDs

$R E \quad J 1036+460, R E J 1043+490, R E \quad J 1126+183$. Three more WDs of our observed sample are known binaries (RE J1036+460 and RE J1126+183, Green, Schmidt, \& Liebert 1986; RE J1043+490, Schwartz et al. 1995) and show red excesses in our spectra: since the $\mathrm{H} \beta$ lines of the WDs are contaminated, they were excluded from the fit. $\mathrm{H} \gamma$ and higher Balmer lines are virtually uncontaminated. A discussion of these stars and newly discovered binaries will be given in a forthcoming paper.

RE J0134-160 (GD 984).- Although our spectrum ends at only $5600 \AA$, a red excess is obvious. Subtraction of the theoretical WD flux leaves an $\mathbf{M}$ star spectrum. The $\mathbf{M}$ dwarf contribution is much smaller than in RE J1629+780, and we excluded only the cores of $\mathrm{H} \beta$ and $\mathrm{H} \gamma$, filled in by the Balmer line emission, from the fit. Bues \& Aslan (1995) suspected a hot third component in RE J0134-160. However, outside of the $\mathrm{H} \beta$ and $\mathrm{H} \gamma$ cores, the Balmer lines are well reproduced by our best fit without any indication of a third component. Indeed, a subdwarf component as suggested by Bues \& Aslan (1995) is almost certainly not present, since its flux would dominate in the blue.

$R E J 1711+664$. - Since a late-type star is separated from the WD by only 2 ..5, we took care to get an uncontaminated WD spectrum. We obtained a spectrum under good seeing conditions. No excess is present up to the red limit at $8500 \AA$.

$R E J 2207+252$. - This WD has a red companion 8.5 away. Schwartz et al. (1995) estimated spectral type K4 V and distance $65 \mathrm{pc}$ from its colors. With the parameters from Table 1, a WD distance of 62 pc results. Thus it is

TABLE 2

RESULTS OF REPEATED OBSERVATIONS

\begin{tabular}{cccccc}
\hline \hline \multirow{2}{*}{$\mathrm{RE}$} & \multicolumn{2}{c}{ OBSERVATION 1} & & \multicolumn{2}{c}{ ObSERVATION 2} \\
\cline { 2 - 3 } \cline { 5 - 6 } \cline { 5 - 6 } & $T_{\text {eff }}$ & $\log g$ & & $T_{\text {eff }}$ & $\log g$ \\
\hline $1235+233$ & $46308 \pm 837$ & $7.85 \pm 0.08$ & & $46737 \pm 670$ & $7.82 \pm 0.06$ \\
$1257+220$ & $39349 \pm 317$ & $7.76 \pm 0.04$ & & $38820 \pm 159$ & $7.78 \pm 0.02$ \\
$1643+411$ & $28813 \pm 120$ & $8.23 \pm 0.02$ & & $28818 \pm 111$ & $8.21 \pm 0.02$ \\
$1650+403$ & $37798 \pm 245$ & $7.94 \pm 0.04$ & & $39126 \pm 413$ & $8.01 \pm 0.05$ \\
$1726+583$ & $52712 \pm 927$ & $8.27 \pm 0.06$ & & $54003 \pm 669$ & $8.20 \pm 0.05$ \\
$2116+735$ & $50131 \pm 384$ & $7.71 \pm 0.03$ & & $54604 \pm 906$ & $7.76 \pm 0.06$ \\
\hline
\end{tabular}

NoTE.-Error estimates were taken from the $\chi^{2}$ fitting procedure. 


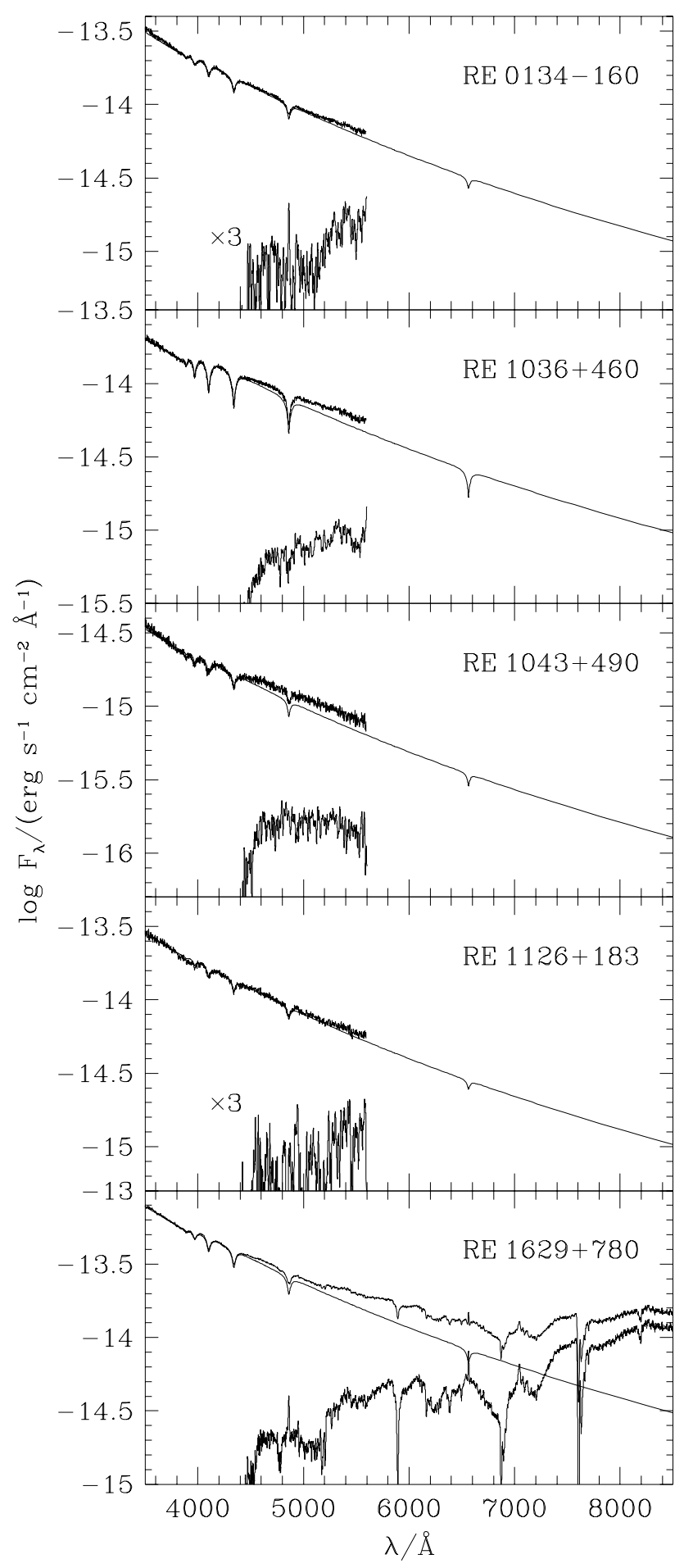

FIG. 5.-Flux-calibrated spectrum of the DA plus $M$ pairs RE $\mathrm{J} 0134-160$, RE $1036+460$, RE J1043+490, RE J1126+183, and RE $\mathrm{J} 1629+780$. From top to bottom we show the composite spectrum, the best fit of the WD, and the resulting spectrum of the $M$ star. The spectrum of the M star companions of RE J0134-160 and RE J1126+183 are multiplied by 3 .

likely that both stars form a physical pair. The angular separation corresponds to $\approx 500 \mathrm{AU}$.

\subsection{Magnetic $W D s$}

Two WDs of our sample show Zeeman splitting of the Balmer line cores, indicative of a magnetic field. RE
$\mathrm{J} 1659+440$ (PG 1658+441) is a well-known star, already analyzed by Schmidt et al. (1992). The discovery of the magnetic nature of RE J1440+750 (HS $1440+7518$ ) was announced by Dreizler et al. (1994; note the naming confusion corrected in Dreizler, Werner, \& Heber 1995-HS $1412+6115$ should have been HS $1440+7518$ ). Although RE J1440+750 was analyzed by V97, they did not remark on its magnetic nature. This is likely due to the lack of coverage of the $\mathrm{H} \alpha$ line, which displays the most pronounced Zeeman effect.

Flux-calibrated spectra of the magnetic WDs are displayed in Figure 6. The PG $1658+441$ analysis of Schmidt et al. (1992) resulted in $T_{\text {eff }}=30,500 \mathrm{~K}$ and $\log g=9.35$. The magnetic splitting was best reproduced by a $3.5 \mathrm{MG}$ dipole inclined $60^{\circ}$ to the line of sight (producing a mean surface field strength $B_{S}=2.3 \mathrm{MG}$ ). From the linear Zeeman effect we estimated a mean magnetic strength of 8 MG for RE J1440+750, consistent with the estimate given in Dreizler et al. (1994).

The temperature and gravity of RE $\mathrm{J} 1440+750$ were derived from a fit of the higher Balmer lines $\mathrm{H} \gamma$ to $\mathrm{H} \eta$ displayed in Figure 7, which are less affected by the magnetic splitting. Results are given in Table 3 supplemented by the Schmidt et al. (1992) fit of PG $1658+441$. Our own fit gave similar results, albeit with lower accuracy. Our fits of RE $\mathrm{J} 1440+750$ can only provide a rough estimate of the stellar parameters. Accurate results can only be expected from a detailed treatment of the magnetic effects.

\subsection{Internal, External, and Systematic Errors}

We have now presented the results of a homogeneous analysis of a sample of 46 hot, EUV-selected WDs based on Balmer line fitting. Three other large samples analyzed with the same method were recently published by M97, V97, and FKB97. Since considerable overlap exists between all four samples, this allows a direct check for systematic errors and the individual scatter on a star by star basis for WDs hotter than $25,000 \mathrm{~K}$.

Since in contrast to previous works our analysis is based on NLTE model atmospheres, we applied the correction vectors given in Figure 3 to correct for the LTE assumption. Since for the hottest WDs these corrections become large while the accuracy of temperature and gravity estimates decreases for both LTE and NLTE analyses, one should exclude comparison of stars with $T_{\text {eff }}>70,000 \mathrm{~K}$. This does not affect our sample, which has a maximum temperature closer to $54,000 \mathrm{~K}$.

Differences (after correction to NLTE) in $T_{\text {eff }}$ and $\log g$ between studies for stars in common with M97, V97, and FKB97 are displayed in Figure 8 as a function of $T_{\text {eff }}$. The magnetic WDs and the binaries, which show significant contamination of the WD spectrum by the companion, are excluded. One can now focus on systematic differences between pairs of studies, e.g., our results (NGS) versus FKB97 (NGS-FKB97), V97-NGS, M97-NGS, FKB97M97, M97-V97, and so on. However, we chose another approach and performed an optimization that simultaneously took into account all values from all stars in common between any samples. In other words, the values given for the systematic differences between our study and the M97, V97, and FKB97 samples form a system for direct transformation between, say, FKB97 and the three other samples. The running averages computed this way are plotted in Figure 8 as a solid line. The actual value was 


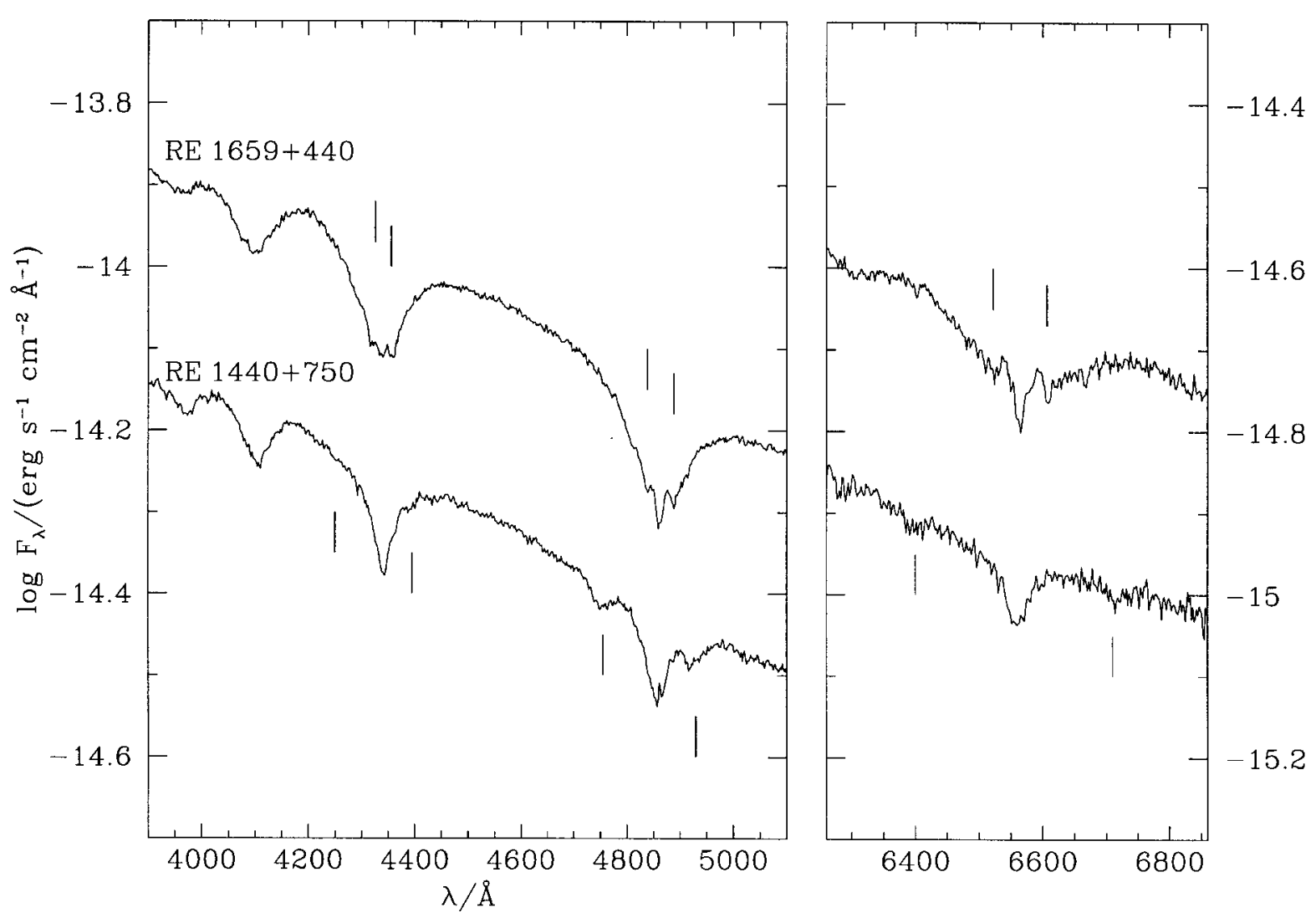

FIG. 6. - Spectra of the magnetic WDs PG $1658+441$ and RE J1440+750. The position of the $\sigma$ components of the Balmer lines are indicated.

computed in $1000 \mathrm{~K}$ steps for all WDs in the respective temperature intervals $T_{\text {eff }} \pm 10 \%$. Although this curve does not represent the best fit to the data plotted in Figure 8 alone, it is a fairly good representation of the differences

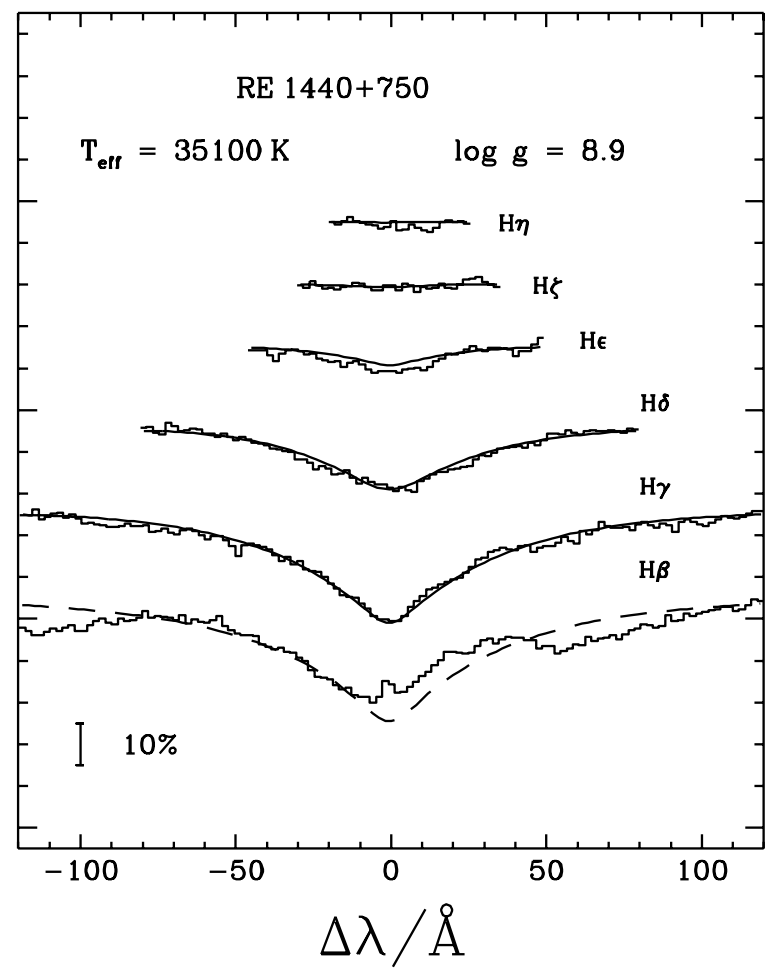

FIG. 7.-Best fit of the magnetic WD RE J1440+750. As indicated by the dashed line, $\mathrm{H} \beta$ was not used for the parameter determination. computed directly between our measurements and those of M97, V97, and FKB97.

Since the distribution is highly non-Gaussian with many outliers, as evident in Figure 8, we decided to adopt an underlying Lorentzian (or Cauchy) distribution for the optimization. The tails of the Cauchy distribution are much larger than that of the corresponding Gaussian, yielding a much lower weight for deviant points (see discussion in Press et al. 1986). The dotted lines represent the $1 \sigma$ confidence interval of the mean, computed conservatively from the rms deviations.

First, we notice a considerable scatter larger than expected from the internal error estimates (see the discussion below). If one ignores the hot end, the agreement between the FKB97 and our temperature scale is good; differences are below $1 \%$, smaller than the maximum model differences to the Koester models (see $\S 4$ ), which were used by FKB97. The same atmospheres are used in M97, and it is therefore surprising that significant differences with M97 are present. These trends are most likely caused by different reduction and analysis techniques. Offsets of the same order are found in our comparison with V97, where a different LTE model atmosphere code is used. Although basically the same input physics is included, this might at least partly explain those shifts in $T_{\text {eff }}$ and $\log g$.

Our results are quantified in Tables 4 and 5 . We divided the WDs into three groups according to their temperature: a cool group with $T_{\text {eff }}<30,000 \mathrm{~K}$, a hotter one with 30,000 $\mathrm{K}<T_{\text {eff }}<45,000 \mathrm{~K}$, and the hottest considered group with $45,000 \mathrm{~K}<T_{\text {eff }}<70,000 \mathrm{~K}$. NLTE effects are still negligible in the range of effective temperatures of the two coolest groups. Mean shifts and the confidence range of the mean (computed as described above) are provided for these 
TABLE 3

PARAMETERS OF THE MAGNeTIC White DWARFS

\begin{tabular}{ccccccc}
\hline \hline \multicolumn{1}{c}{ RE } & Other Names & $T_{\text {eff }}$ & $\log g$ & $M / M_{\odot}$ & $B_{S}$ & Observation Date \\
\hline $1440+750$ & HS $1440+7518$ & $36154 \pm 275$ & $8.87 \pm 0.05$ & 1.128 & 7.7 & 1996 May 10 \\
$1659+440^{\mathrm{a}}$ & PG $1658+441$ & $30510 \pm 200$ & $9.36 \pm 0.07$ & 1.311 & 2.3 & 1996 May 10 \\
\hline
\end{tabular}

${ }^{a}$ Parameters from Schmidt et al. 1992.

groups and the complete sample (except stars with $T_{\text {eff }}>$ $70,000 \mathrm{~K}$ ). The shifts discussed above and shown in Figure 8 are statistically significant and reach values of $\approx 5 \%$ in $T_{\text {eff }}$ in the hottest bin. In $\log g$ the difference between our results and M97 reach $\approx 0.1$ dex for the coolest bin. However, we emphasize that all four analyses are based on state-of-theart model atmospheres and $\chi^{2}$ fitting techniques. Since there are no strong arguments to favor or discard one analysis, it seems these are the systematic shifts characteristic of modern analyses of hot WDs.

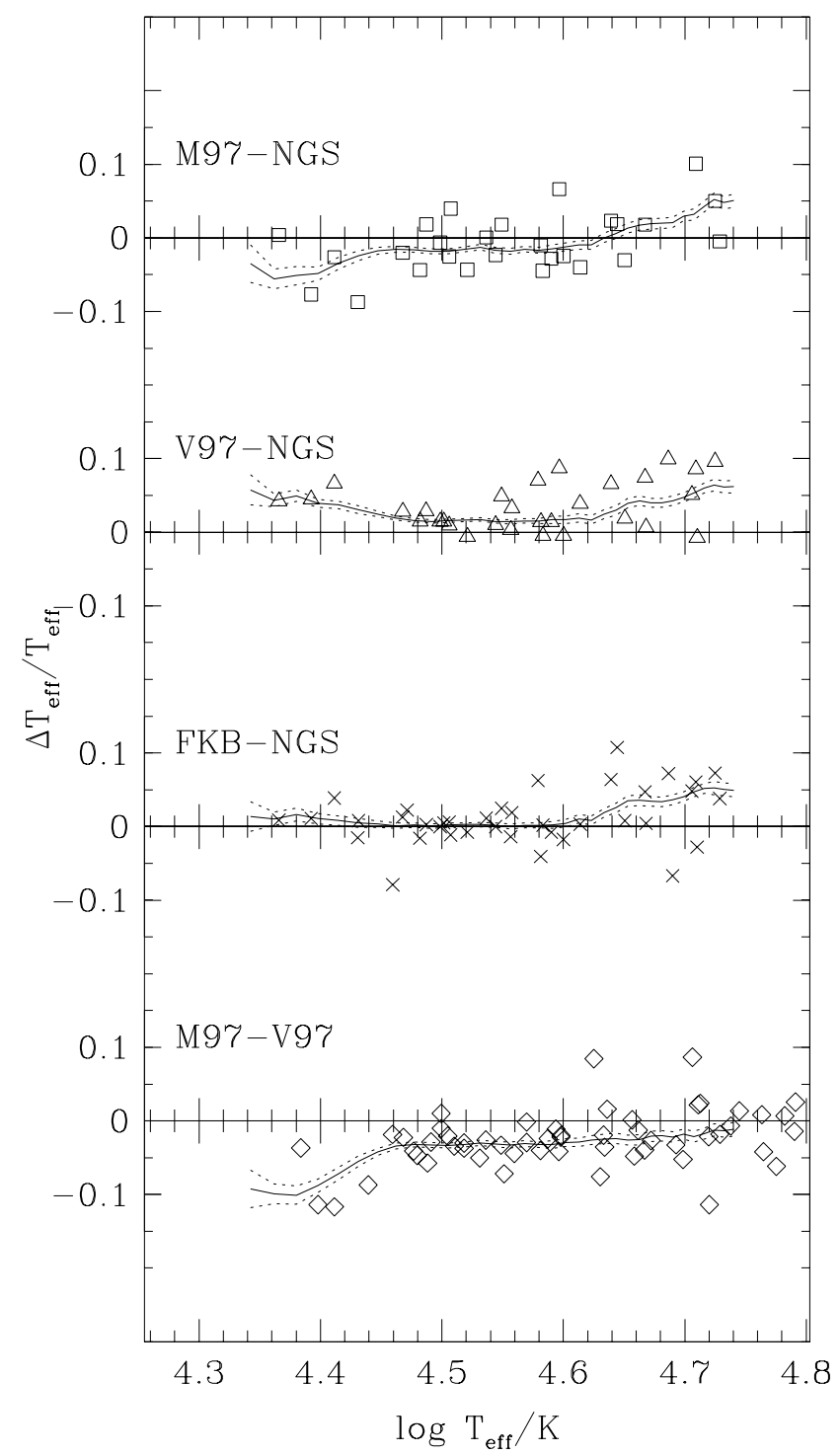

If we take the systematic shifts into account, we can use the samples to derive estimates of the observational scatter, which can be compared with the internal error estimates. Since we have approximately the same scatter for different combinations of the samples, we compute a mean scatter $\sigma_{\text {diff }}$ for all possible combinations (weighted by the number of stars in common). For this purpose we correct for the systematic shifts calculated for each of the three $T_{\text {eff }}$ bins, whose temperature intervals are given in Table 6. The individual measurement errors $\sigma_{\text {ind }}$ add quadratically, and if we

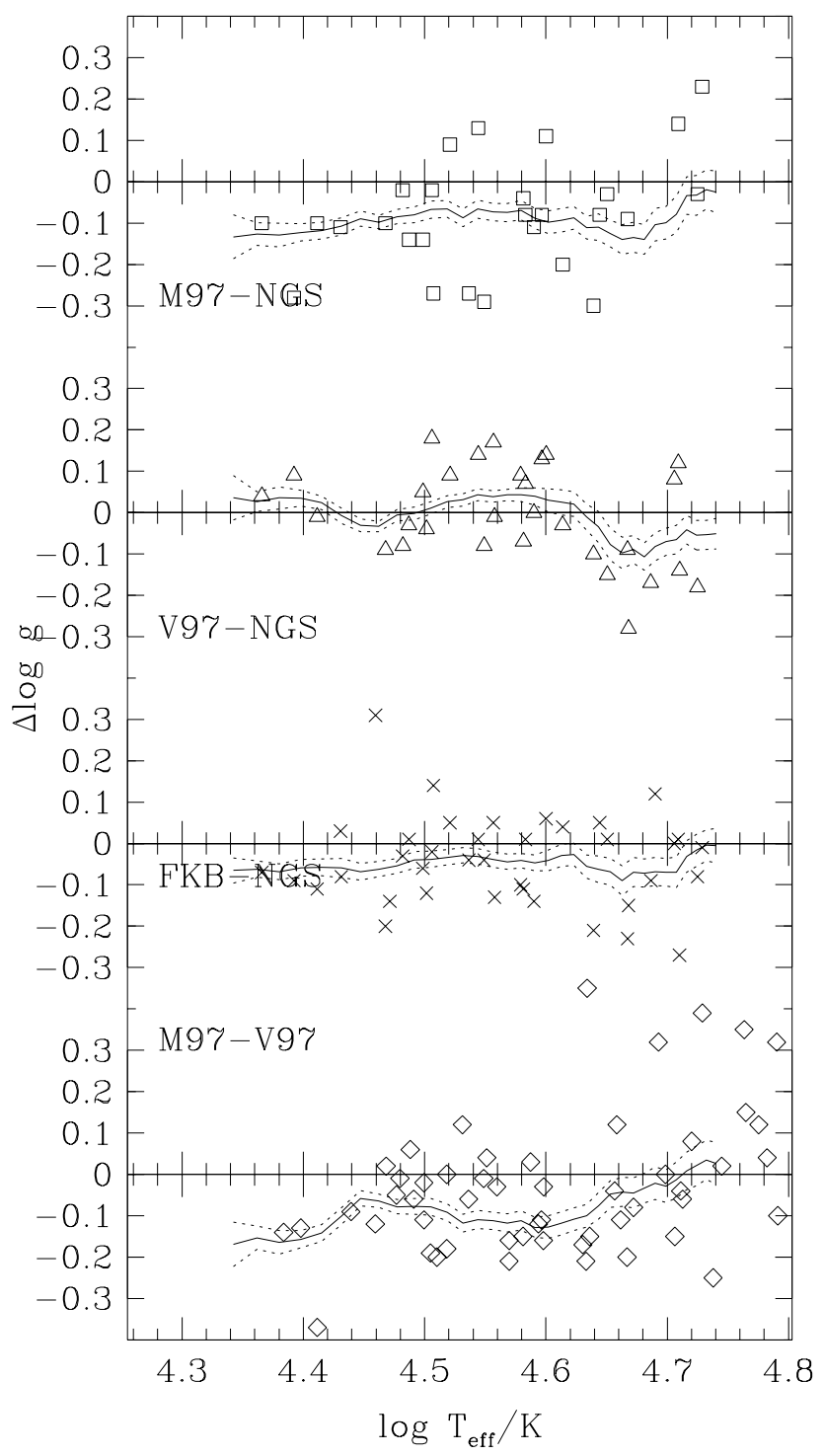

FIG. 8.-Differences in temperature (left-hand panel), and gravity (right-hand panel) between the M97, V97, FKB97 samples, and our own on a star by star basis. The same comparison is carried out for the LTE studies of M97 and V97 in the bottom panel. The smoothed average of the differences is plotted with solid lines, while the standard error of the mean difference is indicated by the dotted lines. The $T_{\text {eff }}$ values used for the $x$-axis are NLTE from this paper, except for the bottom panel, which uses V97 (corrected to NLTE). 
TABLE 4

SYSTEMATIC TEMPERATURE DifFERENCES

\begin{tabular}{cccc}
\hline \hline $\begin{array}{c}\text { Temperature } \\
(\mathrm{K})\end{array}$ & $\begin{array}{c}\text { M97-NGS } \\
\left(\Delta T_{\text {eff }} / T_{\text {eff }}\right)\end{array}$ & $\begin{array}{c}\text { V97-NGS } \\
\left(\Delta T_{\text {eff }} / T_{\text {eff }}\right)\end{array}$ & $\begin{array}{c}\text { FKB97-NGS } \\
\left(\Delta T_{\text {eff }} / T_{\text {eff }}\right)\end{array}$ \\
\hline$T_{\text {eff }}<30000$ & $-0.023 \pm 0.006$ & $0.032 \pm 0.005$ & $0.007 \pm 0.005$ \\
$30000<T_{\text {eff }}<45000$ & $-0.013 \pm 0.003$ & $0.017 \pm 0.003$ & $0.004 \pm 0.003$ \\
$45000<T_{\text {eff }}<70000$ & $0.036 \pm 0.006$ & $0.050 \pm 0.006$ & $0.046 \pm 0.007$ \\
All with $T_{\text {eff }}<70000$ & $-0.006 \pm 0.003$ & $0.024 \pm 0.003$ & $0.012 \pm 0.003$ \\
\hline
\end{tabular}

NoTE.-For each temperature range, each column gives the average difference over the respective interval and the confidence interval of the average difference.

TABLE 5

SYSTEMATIC GRAVITY DIFFERENCES

\begin{tabular}{cccc}
\hline \hline $\begin{array}{c}\text { Temperature } \\
(\mathrm{K})\end{array}$ & $\begin{array}{c}\text { M97-NGS } \\
(\Delta \log g)\end{array}$ & $\begin{array}{c}\text { V97-NGS } \\
(\Delta \log g)\end{array}$ & $\begin{array}{c}\text { FKB97-NGS } \\
(\Delta \log g)\end{array}$ \\
\hline$T_{\text {eff }}<30000$ & $-0.112 \pm 0.017$ & $-0.001 \pm 0.016$ & $-0.064 \pm 0.018$ \\
$30000<T_{\text {eff }}<45000$ & $-0.086 \pm 0.017$ & $0.026 \pm 0.016$ & $-0.034 \pm 0.016$ \\
$45000<T_{\text {eff }}<70000$ & $-0.066 \pm 0.030$ & $-0.067 \pm 0.026$ & $-0.062 \pm 0.027$ \\
All with $T_{\text {eff }}<70000$ & $-0.084 \pm 0.013$ & $-0.002 \pm 0.012$ & $-0.047 \pm 0.012$ \\
\hline
\end{tabular}

NoTE.-For details see Table 4.

assume that the inherent scatter is the same for all analyses (and we found no reason to discard this assumption), the individual measurement errors can be estimated as $\sigma_{\text {ind }}=$ $\left(1 / 2^{1 / 2}\right) \sigma_{\text {diff }}$. Not surprisingly, the smallest scatter is found for the "cool" group $\left(T_{\text {eff }}<30,000 \mathrm{~K}\right)$ with $\sigma\left(T_{\text {eff }}\right)=2.3 \%$ and $\sigma(\log g)=0.07$ dex. It increases to $\sigma\left(T_{\text {eff }}\right)=3.3 \%$ and $\sigma(\log g)=0.13$ dex for the hottest bin. This trend is expected from the statistical analysis presented in Fig. 1 of FKB97. However, the values are larger by a factor of 3 or more than the internal parameter errors for a well-exposed spectrum (see, e.g., Table 1). Therefore, we conclude that the accuracy is not limited by the noise for good spectra, and we suggest that other effects, such as details of the extraction or fluxing and normalization procedures, contribute more. Considering these systematic uncertainties, the 0.3 dex difference between the gravity determinations of FKB97 and Napiwotzki et al. (1993) for HZ 43A is only a $1.5 \sigma$ deviation and therefore not as serious as considered by FKB97.

\subsection{Mass Distribution \\ 5.4.1. Derivation and Sample Comparisons}

Once the temperature and gravity of the WDs are known, the mass can be determined from theoretical mass-radius relations. The recent investigations of M97, V97, and FKB97 based their interpretation on the model sequences

TABLE 6

\begin{tabular}{|c|c|c|}
\hline $\begin{array}{r}\text { MEAN SCATTER } \sigma_{\text {ind }} \\
\text { Gravity DE }\end{array}$ & $\begin{array}{l}\text { TEMPER } \\
\text { MINATI }\end{array}$ & RE AND \\
\hline $\begin{array}{l}\text { Temperature } \\
\text { (K) }\end{array}$ & $\sigma\left(T_{\text {eff }}\right)$ & $\sigma(\log g)$ \\
\hline$T_{\text {eff }}<30000$ & 0.023 & 0.075 \\
\hline $30000<T_{\text {eff }}<45000$ & 0.023 & 0.101 \\
\hline $45000<T_{\text {eff }}<70000$ & 0.034 & 0.128 \\
\hline All with $T_{\text {eff }}<70000$ & 0.026 & 0.107 \\
\hline
\end{tabular}

of Wood (1995). The applied models have $\mathrm{H}$ - and He-layer masses of $10^{-4} M_{\mathrm{WD}}$ and $10^{-2} M_{\mathrm{WD}}$, respectively (thick layers), and a carbon core. These model sequences are based on a set of pre-WD models with end masses of $0.6,0.8$, and $0.95 M_{\odot}$ computed by Kawaler (see Wood 1994). Starting models for other masses were constructed by homology transformations.

The mass-radius relations we use are based on the evolutionary calculations of Blöcker (1995). These models are calculated $\mathrm{ab}$ initio from the main sequence. The resulting WDs show important differences compared to Wood's (1995) models. In contrast with the carbon core models widely used for mass determination, the degenerate core contains a mass-dependent mixture of carbon and oxygen (and trace elements). However, note that Wood (1995) also presented some WD sequences with $\mathrm{C} / \mathrm{O}$ cores. The $\mathrm{H}-$ and He-layer masses resulting from evolutionary calculations depend on the stellar mass (Blöcker 1995; Blöcker et al. 1997). While the canonical thick hydrogen layer mass of $10^{-4} M_{\odot}$ is met for a $0.6 M_{\odot} \mathrm{WD}$, it decreases from several $10^{-3} M_{\odot}$ for $0.3 M_{\odot}$ down to $10^{-6} M_{\odot}$ for $1.0 M_{\odot}$. This results in a mass-radius relation, which is steeper than a relation based on a constant layer mass. Consequently, the derived mass distribution will be narrower if evolutionary layer masses are used.

The lower mass limit for a $\mathrm{C} / \mathrm{O}$ core WD is $0.46 M_{\odot}$, the limiting mass for central helium burning in low-mass stars (Sweigart, Greggio, \& Renzini 1990). Thus WDs with a lower mass possess a helium core. At the current age of the universe He WDs have not been produced by single star evolution, but are the result of binary evolution where the hydrogen-rich envelope was stripped away along the (first) red giant branch (Kippenhahn, Kohl, \& Weigert 1967; Iben $\&$ Tutukov 1986). We use the recent evolutionary models of He WDs calculated by Driebe et al. (1998) based on this scenario.

The combined tracks of Blöcker (1995) and Driebe et al. (1998) cover the mass range $0.18-0.94 M_{\odot}$. We supplement- 
ed this set with the 1.0, 1.1, and $1.2 M_{\odot}$ carbon core sequences of Wood (1995) with "thin" layers. Since the hydrogen envelope mass decreases with increasing WD mass in Blöcker's (1995) models, their highest mass models effectively correspond to thin layer models. Since high-mass WDs are already close to the zero-temperature configuration, the remaining departures from consistent evolutionary calculations can be ignored for practical purposes.

The position of the analyzed WDs in the temperature/ gravity plane is shown in Figure 9 along with the tracks used for the mass determination. The individual WD masses are given in Table 1 , and the resulting mass distribution is shown in Figure 10. We have also redetermined masses for the BSL92 sample with the Blöcker/Driebe mass-radius relations and supplemented Figure 10 with the resulting distribution.

Our mass distribution possesses the same basic features as found in the EUV-selected samples of M97, V97, and FKB97. The sharp peak centered at $\approx 0.59 M_{\odot}$ is in principal agreement with the earlier investigations of KSW79, Weidemann \& Koester (1984), and BSL92, with a sharp falloff toward lower masses and a less steep decline toward higher masses with a tail of high-mass WDs. Considering the relatively large observational scatter we found in $\S 5.3$, the underlying distribution may be extremely sharp. The mean mass is $0.67 M_{\odot}$. However, this value is strongly biased by the few very high-mass WDs.

We decided to follow the recipe of FKB97 and fitted the mass peak with a Gaussian. Although a Gaussian is not a good representation of the WD mass distribution, it gives a robust estimate of the peak mass. The fit result is, in principle, dependent on the adopted binning of the mass intervals. However, in some test calculations we found effects exceeding a few $0.001 M_{\odot}$ only if the bin width was larger than $0.05 M_{\odot}$. The values given in this paper were derived by fitting Gaussians to virtually unbinned mass distributions (formal bin width $0.001 M_{\odot}$ ).

We were concerned by the possibility that the different fraction of low- and high-mass WDs found in different samples (see discussion below) may skew the Gaussian fit of the main peak to higher or lower masses. We tested this by fitting the BSL92, FKB97, and our mass distributions with multicomponent Gaussians, which fitted the secondary high- and low-mass peaks separately. We found no deviation higher than $0.003 M_{\odot}$ in any case and concluded that the single Gaussian fit of the main peak yields a rather stable estimate.

With this method, we derived a peak mass of our sample of $0.589 M_{\odot}$. We reanalysed the FKB97, M97, and V97 samples with our mass-radius relations. We applied the cor-

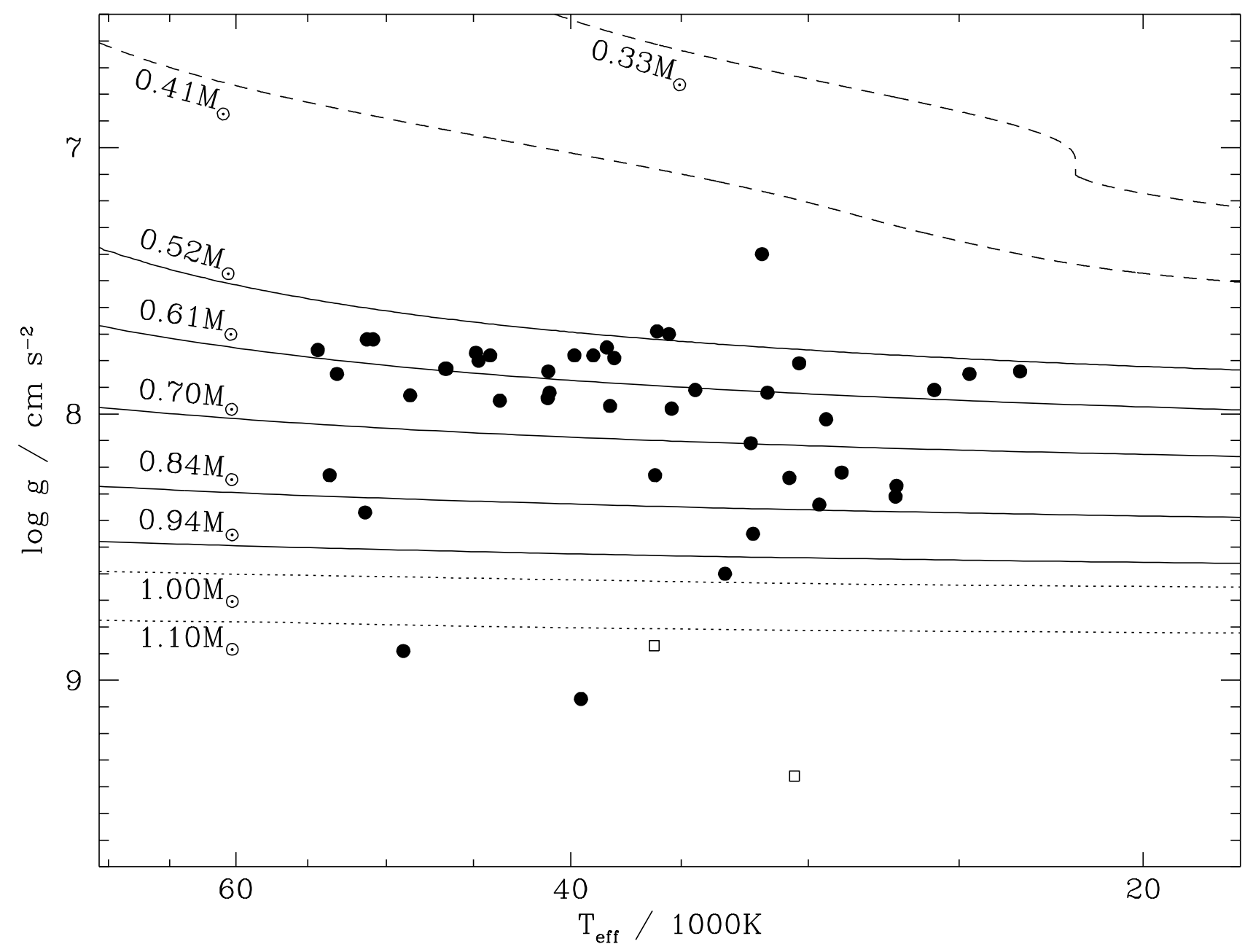

FIG. 9.-Effective temperature and gravity of our WD sample compared with evolutionary tracks. Solid lines are Blöcker (1995); dashed lines are Driebe et al. (1998); dotted lines are Wood (1995). The magnetic WDs are marked by open symbols. 


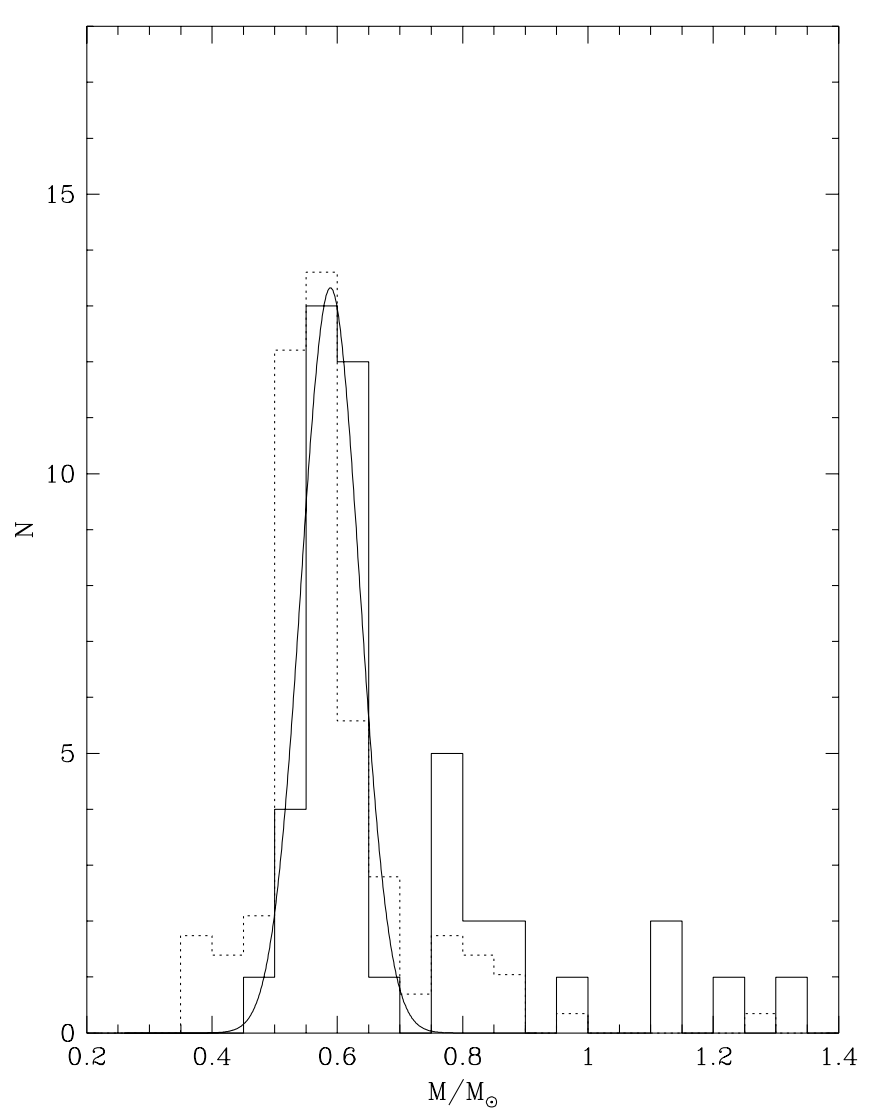

FIG. 10.-Mass distribution of WDs (binned over $0.05 M_{\odot}$ ). The solid line histogram indicates the result for our sample. The dotted line is the BSL92 sample with masses redetermined from the Blöcker/Driebe relations (normalized to the same sample size). The Gaussian curve represents the best fit to our mass distribution as described in the text.

rections to NLTE and excluded the WDs with temperatures in excess of 70,000 K. The peak masses are 0.555 (FKB97), 0.535 (M97), and $0.582 M_{\odot}$ (V97). These differences reflect the systematic differences in $T_{\text {eff }}$ and $\log g$ determinations discussed above, and probably different selection criteria in the case of FKB97.

The probable He core WD RE J0512-004 is the only object in our sample with a mass below $0.5 M_{\odot}$, but four WDs have masses in excess of $1.0 M_{\odot}$. The frequency of low- and high-mass WDs found in our and other EUVselected samples is qualitatively different from that found in optically selected samples such as BSL92. BSL92 detected in their sample of 129 WDs 16 objects with masses below the limiting mass for $\mathrm{C} / \mathrm{O}$ core WDs and a total of $28 \mathrm{WDs}$ with $M<0.5 M_{\odot}$. These numbers are reduced to 10 and 16, respectively, if we redetermine the masses with the massradius relations of Blöcker (1995) and Driebe et al. (1998). However, that is still a much higher fraction than we find in our EUV-selected sample.

\subsubsection{Cooling Rates and Detection Probability}

Why do optical- and EUV-selected samples contain different fractions of high- and low-mass WDs? All helium WDs from the BSL92 sample have temperatures below $30,000 \mathrm{~K}$; the only helium WD candidate in our sample has $T_{\text {eff }} \approx 32,000 \mathrm{~K}$. This implies that the detection probability of low-mass WDs in EUV-selected samples is much lower, because the fraction of low-mass WDs at higher $T_{\text {eff }}$ is much lower than it is for $T_{\text {eff }}<30,000 \mathrm{~K}$. This viewpoint is supported by the helium WD sequences calculated by Driebe et al. (1998). We have plotted the variation of temperatures with time for WDs with helium and C/O cores in Figure 11. The detection probability in a given evolutionary stage goes as the inverse of the rate of temperature decrease at that stage. New He WDs cool down rapidly to a certain (massdependent) temperature, whereafter the cooling rate drops drastically and the star remains on a temperature plateau for a long time. This behavior is most pronounced for the lowest masses. Hydrogen shell burning plays an important role for this plateau phase. Although the energy production by hydrogen burning drops down dramatically after the star enters the WD sequence, it still produces a significant fraction of the total luminosity, enough to bring the cooling to a standstill. We can conclude from Figure 11 that it is extremely unlikely to detect a helium WD during its first rapid cooling phase; it is easier to find them during their plateau phase or afterward. This means there is a strong bias toward detecting helium WDs in samples of relatively "cool" DA stars (like BSL92) instead of the overall much hotter EUV-selected samples.

We can explain in a similar fashion why high-mass WDs are found preferentially in the $30,000 \mathrm{~K}<T_{\text {eff }}<50,000 \mathrm{~K}$ range. Due to the drop in neutrino cooling, the evolutionary speeds of massive WDs are relatively low in this range, as demonstrated by the $0.940 M_{\odot}$ track, the most massive stellar remnant calculated by Blöcker (1995). This increases the detection probability of more massive WDs in EUVselected samples.

\subsubsection{Interstellar Absorption}

Even if we restrict our comparisons to hot subsamples of WDs, the fraction of massive WDs is much higher in the EUV-selected samples than it is in optically selected ones. An extensive discussion of this problem is carried out by FKB97. FKB97 argue that the number of high-mass WDs discovered per sky area is similar for the whole-sky EUV surveys and the optical Palomar-Green (PG) survey (Green et al. 1986), which covered roughly $25 \%$ of the sky. In other words, a whole-sky version of the PG survey should have discovered all but one of the EUV-detected WDs with $M>1.1 M_{\odot}$. At a given temperature, WDs with lower masses are larger, consequently more luminous, and detectable to larger distances. For instance, at $30,000 \mathrm{~K}$ a WD of $0.5 M_{\odot}$ is detectable at distances $\sim 30 \%$ larger than a 0.7 $M_{\odot}$ WD. At the maximum distance sampled by, e.g., the PG survey, the probability is very high that interstellar matter effectively absorbs all EUV radiation. Thus the dominant effect in EUV surveys is not a selection for massive WDs as suggested by V97, but a selection against low-mass WDs due to a sample volume strongly affected by interstellar absorption.

\subsubsection{Temperature Dependence of the Derived Mass Peak}

FKB97 noted a moderate apparent trend of the WD peak mass with temperature. Since their sample also included optically selected WDs, they analyzed a considerably higher fraction of stars with $T_{\text {eff }}<25,000 \mathrm{~K}$, and therefore have a larger temperature baseline than pure EUV samples. Analogous to this finding, V97 found an $0.03 M_{\odot}$ offset between the peak of the mass distribution in their EUV-selected sample and the (cooler) WDs analyzed by BSL92. In both 


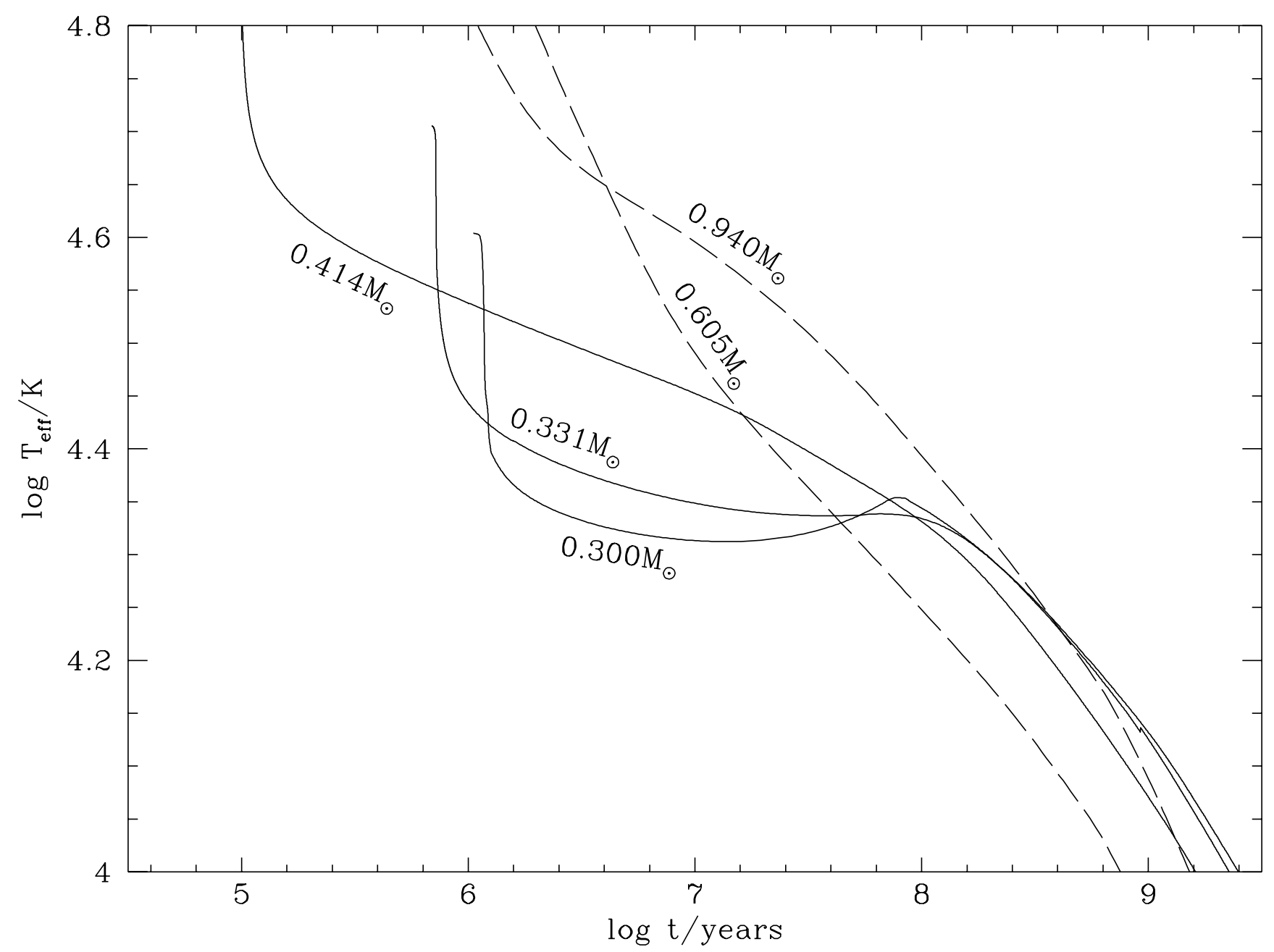

FIG. 11.-Time evolution of stellar surface temperature for helium core WDs (tracks from Driebe et al. 1998; solid lines) and C/O WDs (tracks from Blöcker 1995; dashed lines). The tracks are labeled with the stellar masses.

cases this temperature dependence decreases if Wood's (1995) models with very thin layers $\left(M_{\mathrm{He}}=10^{-4} M_{\mathrm{wD}}\right.$, no hydrogen layer), instead of the canonical thick layer models $\left(M_{\mathrm{He}}=10^{-2} M_{\mathrm{wD}}, M_{\mathrm{H}}=10^{-4} M_{\mathrm{wD}}\right)$, are used. However, part of the trend noted in V97 may be related to the systematic differences between the analyses discussed in $\S$ 5.3. Since V97 compared results from two different analyses, it is not possible to judge if the offset is real or just an artifact. The case of FKB97 is stronger, because they based their case on a homogeneously analyzed sample. FKB97 warned that the temperature dependence of the mass distribution peak may be caused by inadequacies remaining in the model atmospheres. A more detailed discussion is given in the next section.

Some evidence that part of the temperature trend stems from the analyses comes from our intercomparisons presented in $\S 5.3$. We showed that systematic differences between the four investigated samples exist that vary with effective temperature. This might mimic a temperature dependence of the sample peak mass. A gravity offset of 0.1 dex transforms into mass offsets of $0.050,0.044$, and 0.034 $M_{\odot}$ for a $0.6 M_{\odot}$ WD with $25,000,40,000$, and $60,000 \mathrm{~K}$, respectively. Even a relatively small systematic $\log g$ difference of $0.05 \mathrm{dex}$ (see Table 5) corresponds to a $0.02 M_{\odot}$ offset. The scatter $\sigma$ of individual gravity determinations reported in Table 6 corresponds to $\sigma(M) \approx 0.04 M_{\odot}$, nearly independent of $T_{\text {eff }}$.

\subsubsection{Cooling Tracks}

Another part of the $T_{\text {eff }}$ dependence of the mass peak may be caused by the use of Wood's (1995) cooling tracks by FKB97 and V97. These tracks are not completely selfconsistent since not all model sequences were not calculated $\mathrm{ab}$ initio from the main sequence. This is not important for cooler WDs, but it may cause deviations from ab initio tracks, especially for the hottest WDs where structure depends sensitively on the evolutionary history (Blöcker \& Schönberner 1990).

We redetermined the masses of the BSL92 WDs with the Blöcker/Driebe mass-radius relations and derived a peak mass of $0.559 M_{\odot}$. That is $0.03 M_{\odot}$ lower than our peak mass, but having in mind the range of mass determination derived for the EUV-selected samples, we cannot consider this a significant difference. FKB97 divided their sample into cool $\left(T_{\text {eff }}<35,000 \mathrm{~K}\right)$ and hot $\left(35,000 \mathrm{~K}<T_{\text {eff }}<\right.$ $75,000 \mathrm{~K})$ subsamples and derived a $0.029 M_{\odot}$ higher peak mass for the hot sample. Our reanalysis of the FKB97 LTE results with the Blöcker/Driebe mass-radius relation yields 
virtually the same offset: $0.025 M_{\odot}$. However, the difference is brought down to $0.010 M_{\odot}\left(0.550\right.$ vs. $\left.0.560 M_{\odot}\right)$ if we apply corrections to NLTE. One can imagine that a difference of this order (if significant at all) can easily be produced by our neglect of metallicity effects (see Lanz et al. 1996; Barstow et al. 1998).

Therefore, given the combination of sample selection and systematic effects in analyses to date, our results do not confirm the presence of intrinsic systematic mass differences between hot and cool WDs.

\section{CONCLUSIONS}

We have obtained temperatures, gravities, and masses for a sample of 46 EUV-selected DA WDs. These data complement a near-IR survey for low-mass companions. The stellar parameters were determined by fitting the hydrogen Balmer line profiles with NLTE model spectra. A map of LTE correction vectors was constructed, which allows the transformation of LTE results to the NLTE scale. These shifts become important for temperatures above $50,000 \mathrm{~K}$ (see also Napiwotzki 1997).

Three recent analyses of DA WDs have a considerable overlap with our sample and allow a direct check for systematic errors and individual measurement scatter. Although all four analyses applied similar model atmospheres and fitting techniques, we recognized systematic shifts up to $5 \%$ in temperature and 0.1 dex in gravity determinations. These systematic errors must be considered when the results are interpreted and compared to other studies. The individual measurement errors increase with temperature: $\sigma\left(T_{\text {eff }}\right)$ from $2.3 \%$ to $3.3 \%$ and $\sigma(\log g)$ from 0.07 to 0.13 dex. This is larger than the typical errors computed with $\chi^{2}$ fit procedures for a well-exposed spectrum, and it indicates that accuracy is limited by noise in combination with other effects such as details of the extraction, fluxing, or normalization procedures. It is unclear if these $\sigma$ values indicate lower limits or scale (at least partially) with the $\mathrm{S} / \mathrm{N}$ of the spectra. It will be a challenge to resolve this question. Repeated observations performed by one observer, at one telescope, reduced the same way, and analyzed with one method as performed by BSL92, FKB97, and us (Table 2) do not reveal all effects. Independent observations analyzed independently are necessary.

Masses have been inferred from theoretical mass-radius relations based on the evolutionary calculations of Blöcker
(1995) for C/O WDs and Driebe et al. (1998) for He WDs. An important feature of these calculations are the hydrogen and helium layer masses, which depend on stellar mass. We find a sharp peak centered at $\approx 0.59 M_{\odot}$ in agreement with the previous investigations of KSW79 and BSL92. We redetermined masses of the BSL92 WDs with the Blöcker/Driebe mass-radius relation and derived a peak mass of $0.56 M_{\odot}$. At first glance this seems to confirm the systematic offset reported by V97. However, this offset could be explained by systematic differences of the model atmosphere analyses as well. Our reanalysis of the homogeneous FKB97 sample showed that the temperature dependence of the mass peak nearly vanishes when corrected for their LTE assumption. We conclude that the observational data presented here and in similar studies are well explained by canonical stellar evolution theory, i.e., WDs with thick envelopes.

We find only one object (RE J0512-004) with a mass below $0.5 M_{\odot}$, which is a possible helium core WD, but four WDs with masses in excess of $1.0 M_{\odot}$. This ratio of highand low-mass WDs is quite different from that found by BSL92. This can partly be understood as the result of variable evolutionary timescales of high- and low-mass WDs. We agree with FKB97 that another part can be explained by a selection against low-mass WDs in EUV-selected samples.

The principal aim of our project is the search for and study of cool main-sequence companions of our WD sample. Five WDs already show red contamination in our optical spectra. A comprehensive discussion of these and other binaries will be given in a forthcoming paper presenting IR photometry for the WD sample. In this article we provided the basic WD data necessary to interpret our binary sample.

We thank T. Driebe, F. Herwig, and T. Blöcker for providing us with their tracks and computing the WD masses, and D. Koester, who made some model spectra available for our model comparison. We are grateful to Steward Observatory for an unexpected generous award of six nights of unclaimed $2.3 \mathrm{~m}$ time, which made possible simultaneous optical and IR observations of our sample of stars. We thank Perry Berlind for obtaining several WD spectra. P. J. G. acknowledges support through NASA contract NAS8-39073 (ASC).

\section{REFERENCES}

Barstow, M. A., Hubeny, I., \& Holberg, J. B. 1998, MNRAS, 299, 520

Beauchamp, A., Wesemael, F., Bergeron, P., Liebert, J., \& Saffer, R. A. 1996, in ASP Conf. 96, Hydrogen-Deficient Stars, ed. C. S. Jeffery \& U. Heber (San Francisco: ASP), 295

Bergeron, P. 1993, in White Dwarfs: Advances in Observation and Theory, ed. M. A. Barstow (Dordrecht: Kluwer), 267

Bergeron, P., Saffer, R. A., \& Liebert, J. 1992, ApJ, 394, 228 (BSL92)

Blöcker, T. 1995, A\&A, 299, 755

Blöcker, T., Herwig, F., Driebe, T., Bramkamp, H., \& Schönberner, D. 1997, in White Dwarfs, ed. I. Isern et al. (Dordrecht: Kluwer), 57

Blöcker, T., \& Schönberner, D. 1990, A\&A, 240, L11

Bues, I., \& Aslan, T. 1995, in White Dwarfs, ed. D. Koester \& K. Werner (Berlin: Springer), 259

Burleigh, M. R., Barstow, M. A., \& Fleming, T. A. 1997, MNRAS, 287, 381

Catalán, M. S., Sarna, M. J., Jomaron, C. M., \& Smith, R. C. 1995, MNRAS, 275, 153

deKool, M., \& Ritter, H. 1993, A\&A, 267, 397

Dreizler, S., Heber, U., Jordan, S., \& Engels, D. 1994, in Hot Stars in the Galactic Halo, ed. S. J. Adelman, A. R. Upgren, \& C. J. Adelman (Cambridge: Cambridge Univ. Press), 228

Dreizler, S., \& Werner, K. 1996, A\&A, 314, 217

Dreizler, S., Werner, K., \& Heber, U. 1995, in White Dwarfs, ed. D. Koester

$\&$ K. Werner (Berlin: Springer), 160
Driebe, T., Schönberner, D., Blöcker, T., \& Herwig, F. 1998, A\&A, 339, 123

Filippenko, A. V. 1982, PASP, 94, 715

Finley, D. S., et al. 1993, ApJ, 417, 259

Finley, D. S., Koester, D., \& Basri, G. 1997, ApJ, 488, 375 (FKB97)

Green, P. J., \& Margon, B. 1994, ApJ, 423, 723

Green, R. F., Schmidt, M., \& Liebert, J. 1986, ApJS, 61, 305

Heber, U., Bade, N., Jordan, S., \& Voges, W. 1993, A\&A, 267, L31

Hubeny, I., Hummer, D. G., \& Lanz, T. 1994, A\&A, 282, 151

Hummer, D. G., \& Mihalas, D. 1988, ApJ, 331, 794

Iben, I., Jr., \& Tutukov, A. V. 1986, ApJ, 311, 742

Jordan, S., Heber, U., \& Weidemann, V. 1991, in White Dwarfs, ed. G.

Vauclair \& E. M. Sion (Dordrecht: Kluwer), 121

Kidder, K. M. 1991, Ph.D. thesis, Univ. Arizona

Kidder, K. M., Holberg, J. B., Barstow, M. A., Tweedy, R. W., \& Wesemael, F. 1992, ApJ, 394, 288

Kippenhahn, R., Kohl, K., \& Weigert, A. 1967, Z. Astrophys., 66, 58

Koester, D., Schulz, H., \& Weidemann, V. 1979, A\&A, 76, 262 (KSW79)

Lanz, T., Barstow, M. A., Hubeny, I., \& Holberg, J. B. 1996, ApJ, 473, 1089

Lemke, M. 1997, A\&AS, 122, 285

Liebert, J., Bergeron, P., \& Saffer, R. A. 1990, PASP, 102, 1126

Malina, R., et al. 1994, AJ, 107, 751

Marsh, M. C., et al. 1997, MNRAS, 286, 369 (M97)

Massey, P., Strobel, S., Barnes, J. V., \& Anderson, E. 1988, ApJ, 328, 315 
Napiwotzki, R. 1997, A\&A, 322, 256

Napiwotzki, R., Barstow, M. A., Fleming, T., Holweger, H., Jordan, S., \& Werner, K. 1993, A\&A, 278, 478

Pounds, K., et al. 1993, MNRAS, 260, 77

Press, W. H., Flannery, B. P., Teukolsky, S. A., \& Vetterling, W. T. 1986, Numerical Recipes (Cambridge: Cambridge Univ. Press)

Schmidt, G. D., Bergeron, P., Liebert, J., \& Saffer, R. A. 1992, ApJ, 394, 603

Schwartz, R. D., Dawkins, D., Findley, D., \& Chen, D. 1995, PASP, 107, 667

Sion, E. M., Holberg, J. B., Barstow, M. A., \& Kidder, K. M. 1995, PASP, 107,232

Sweigart, A. V., Greggio, L., \& Renzini, A. 1990, ApJ, 364, 527

Vennes, S., Christian, D. J., \& Thorstensen, J. R. 1998, ApJ, 502, 763

Vennes, S., Thejll, P. A., Galvan, G. G., \& Dupuis, J. 1997, ApJ, 480, 714 (V97)

Vennes, S., \& Thorstensen, J. R. 1994, ApJ, 433, L29

Vidal, C. R., Cooper, J., \& Smith, E. W. 1970, J. Quant. Spectrosc. Radiat. Transfer, 10, 1011

Weidemann, V., \& Koester, D. 1984, A\&A, 132, 195

Werner, K. 1986, A\&A, 161, 177

Wonnacott, D., Kellett, B. J., \& Stickland, D. J. 1993, MNRAS, 262, 277

Wood, M. 1994, in IAU Colloq. 147, Equation of State in Astrophysics, ed. G. Chabrier \& E. Schatzmann (Cambridge: Cambridge Univ. Press), 612 . 1995, in White Dwarfs, ed. D. Koester \& K. Werner (Berlin: Springer), 41 